\title{
EP300 (p300) mediated histone butyrylation is critical for adipogenesis
}

\author{
Aditya Bhattacharya ${ }^{1}$, Sourav Chatterjee ${ }^{1}$, Utsa Bhaduri ${ }^{2}$, Akash Kumar Singh ${ }^{1}$, Madavan \\ Vasudevan $^{3}$, Koneni V Sashidhara ${ }^{5}$, Rajdeep Guha ${ }^{6}$, Nagashayana Natesh ${ }^{7}$, Tapas K Kundu ${ }^{1,4}$ \\ 1 Transcription and Disease Laboratory, Molecular Biology and Genetics Unit, Jawaharlal Nehru Centre for \\ Advanced Scientific Research, Bangalore, India. \\ 2 Chromatin Biology Laboratory, Molecular Biology and Genetics Unit, Jawaharlal Nehru Centre for \\ Advanced Scientific Research, Bangalore, India. \\ 3 Theomics International Pvt Ltd, Bangalore \\ 4 Division of Neuroscience and Aging Biology,CSIR-Central Drug Research Institute, Lucknow \\ 5 Division of Medicinal and Process Chemistry, Central Drug Research Institute, Lucknow, India \\ 6 Division of Laboratory Animal Facility, Central Drug Research Institute, Lucknow, India \\ 7 Central Government Health Scheme Wellness Centre Number 8, Domlur, Bangalore, India
}

\section{Corresponding author: Tapas K Kundu}

E-mail ID of corresponding author: tapas@jncasr.ac.in

\begin{abstract}
Author Contributions: $\mathrm{AB}$ and TKK conceived the project. $\mathrm{AB}$ designed and performed all the biological experiments. SC performed initial synthesis of all compounds and molecular docking analysis. KVS performed bulk scale synthesis of LTK-14A for animal experiments. AKS and RG assisted in the animal experiments. UB and MV performed in silico analysis of high throughput RNA sequencing data. NN provided the raw material Garcinia indica for compound isolation. AB and TKK analyzed the data and wrote the manuscript.
\end{abstract}

Competing Interest Statement: Authors declare no conflict of interest.

Classification: Major category- Biological Sciences. Minor category- Biochemistry.

Keywords: Keywords: Acetylation / adipogenesis / butyrylation / garcinol / p300

This PDF file includes:

Main Text

Figures 1 to 8

Tables 1 to 7 


\begin{abstract}
The master epigenetic enzyme EP300 (p300) besides having lysine acetyltransferase activity can also catalyse other acylation modifications (propionylation, butyrylation, crotonylation etc.), the physiological implications of which are yet to be established. Here we report that lysine butyrylation concomittantly increases during adipogenesis, along with increased butyryl CoA levels due to upregulated fatty acid metabolic pathways. To delineate the role of p300 catalysed butyrylation in adipogenesis, we have identified a semi-synthetic derivative (LTK-14A) of garcinol which specifically inhibited histone butyrylation without affecting acetylation. Treatment of 3T3L1 cells with LTK-14A significantly abolished adipogenesis by downregulation of genes related to adipogenesis presumably through the inhibition of H4K5 butyrylation. Administering the specific inhibitor to high fat diet fed C57BL6/J mice as well as genetically obese $\mathrm{db} / \mathrm{db}$ mice led to an attenuation/decrease in their weight gain respectively. The reduced obesity could be at least partially attributed to the targeted inhibition of H4K5 butyrylation, as observed by immunofluorescence staining of the inhibitor treated mice liver sections and immunoblotting with histones extracted from epididymal fat pads. This report therefore not only for the first time causally links histone butyrylation with adipogenesis but also presents a novel small molecule modulator that could be developed for anti-obesity therapeutics.
\end{abstract}

\title{
Significance Statement
}

In the modern era, obesity has become a highly prevalent disorder, characterized by adipose tissue hypertrophy. Excessive caloric intake with minimal energy expenditure leads to accumulation of lipids in the adipose tissue. Like in many other processes, adipogenesis is epigenetically regulated. Besides acetyltransferase activity, the epigenetic enzyme p300 also possesses acyltransferase activity such as butyrylation. Our findings not only demonsrate the physiological role of p300 catalysed butyrylation in adipogenesis but also report a selective inhibitor of butyrylation which could have high therapeutic potential.

\section{Main Text}

\section{Introduction}

Chromatin function is spatially and temporally regulated by several processes like incorporation of histone variants, remodeling of nucleosomes by ATP-dependant complexes and post-translational modifications of histones. A further layer of complexity is added to it by post-translational modifications of chromatin associated proteins such as histone chaperones and non-histone chromatin proteins which in turn modulate their activities. Interestingly, epigenetics and the metabolic state of the cell are intimately intertwined because the enzymes catalyzing the modifications use co-factors derived from several metabolic reactions. As for example, the highly studied reversible lysine acetylation modification is critically regulated by the concentration of acetyl group donor acetyl CoA, an intermediate of cellular respiration and fatty acid metabolism (1). Since the last decade, owing to major advances in high sensitivity mass spectrometry, more and more new post-translational modifications are being discovered. Of particular interest is a group of acylation modifications which structurally closely resemble acetylation, but differ in hydrocarbon chain length, hydrophobicity and charge (2). Emerging evidences in recently published articles indicate that these modifications occur dynamically on histones and could either have distinct functions from acetylation, or, have a similar but more profound impact on chromatin decompaction, thereby amplifying the transcriptional output. EP300 (p300) is the master acyltransferase that has been found to promiscuously acylate histones using diverse CoA derivatives. By virtue of the presence of a hydrophobic pocket to accommodate the variable aliphatic portion of the acyl CoA, p300 can catalyze these additional modifications albeit at a slower rate than acetylation (3).

The process of adipogenesis is accompanied by a significant increase in global histone acetylation levels, along with a rise in expression of $\mathrm{p} 300$ and a concomitant decrease in the levels of several histone deacetylases (HDAC 1, HDAC 2, HDAC 5 and HDAC 6) (4). Amongst the histone deacetylases, Sirtuins 1-3 have been found to possess broad range deacylase activities in vitro (5). But recently, it has been shown that HDAC 1 and 2, which form the catalytic core of several repressor complexes can also carry 
out decrotonylation of substrates in embryonic stem cells (6). Regulation of gene expression is dictated by the fluctuating activities of histone acetyltransferases and histone decetylases. Moreover, the importance of histone acetylation for adipogenesis has been further underlined by the finding that knockdown of ATP citrate lyase, an enzyme responsible for regeneration of acetyl CoA in the cytoplasm and nucleus, leads to decrease in histone acetylation and also suppression of adipogenesis (7). Therefore, it can be assumed that p300; an acetyltransferase plays a crucial role in promoting the expression of adipogenesis related genes by acetylating the histones in the corresponding gene regulatory regions, especially the promoters. Amongst the different coenzyme A derivatives, butyric acid is an important short chain fatty acid and an intermediate of fatty acid synthesis/breakdown processes. p300 has been known to carry out butyrylation of histones which has been causally linked to the process of spermatogenesis (8).

In this study we report that histone butyrylation is critical in adipoogenesis, probed by the first known acylation specific small molecule inhibitor of p300. The newly discovered importance of histone butyrylation in adipogenesis makes it an attractive target for small molecule compound mediated inhibition of adipogenesis and thereby controlling obesity with lesser collateral damage.

\section{Results}

Physiological levels of butyryl CoA and histone butyrylation increases during adipogenesis: Under normal circumstances, the cellular levels of certain coenzyme A derivatives are much less than acetyl CoA (9).We surmised that the situation may alter in specific cases where metabolic pathways promoting the production of other acyl CoA derivatives become more predominant. One such possible scenario could be the process of adipogenesis in which even chained fatty acid synthesis and breakdown can give rise to several intermediate products like butyryl CoA.

The possible causal relationship of butyryl CoA and adipogenesis was investigated using the 3T3L1 preadipocyte cell line as model system. The cells were subjected to adipogenesis process and on seventh day it was confirmed by Oil Red-O staining (fig. 1 a). The expression of the molecular markers of adipogenesis such as Pparg and Cebpa further confirmed the differentiation process (fig. 1 b). Interestingly, a significant increase in the expression of Acss2 (acyl-CoA synthetase short chain family member 2) upon differentiation could also be observed. Previously, ACSS2 had been established as the enzyme responsible for generation of acyl-CoA derivatives from their corresponding short chain fatty acids (10).

In order to investigate the metabolic state (especially concentration of butyryl CoA) of the cells during differentiation, metabolites were extracted from undifferentiated and differentiated 3T3L1 cells and the stoichiometric levels of butyryl CoA were estimated by mass spectrometry analysis (Sup fig. $1 \mathrm{a}, \mathrm{b}, \mathrm{c}$ ). A near two-fold increase in the levels of butyryl CoA was observed upon differentiation (Sup fig. $1 \mathrm{~d}, e$ ). The enhanced transcript level of Acss2 and stoichiometric level of butyryl CoA suggested that ACSS2 could possibly play a critical role in increased conversion of butyric acid to butyryl CoA, which in turn may lead to a more pronounced physiological role of butyryl CoA during adipogenesis. These observations prompted us to investigate whether this also led to an increased butyrylation of proteins. For this purpose, histones were acid extracted from undifferentiated and differentiated 3T3L1 cells followed by immunoblotting. We observed that in accordance with previous report, there was indeed an increase in histone acetylation level (H3K9 Ac) in the differentiated cells as compared to the undifferentiated cells (Fig. 1 c, panel i). Interestingly, we also observed enhanced global histone butrylation levels upon differentiation. Immunoblotting analysis with site-specific antibodies against histone butyrylation marks (H3K9, H3K23, H3K27, H4K5, H4K8 and H4K12 Butyrylation) (Fig. 1c, panel ii and iii) also revealed that butyrylation at each of these sites increased upon differentiation. Amongst these modifications, H4K8 butyrylation was barely detectable in the undifferentiated pre-adipocytes and showed a dramatic increase in adipocytes. Collectively these results showed that during adipogenesis, EP300 catalysed butyrylation of histones could be an important epigenetic signature.

\section{Identification of a small molecule that selectively inhibits p300 catalysed histone butyrylation in vitro:}

Butyrylation is an acylation reaction which can be catalysed by different lysine acetyltransferases (KATs) with varying efficiency. Amongst the different KAT families, the EP300 belonging to the p300/CREB binding protein (p300/CBP) family has emerged as the most promiscuous acyltransferase. Unlike the 
other enzymes, EP300 possesses a deep aliphatic pocket within which the acyl chains of different coenzyme A derivatives can fit inside, thereby enabling the transfer of the acyl group onto the nucleophilic lysine residue (3). We therefore targeted EP300 to get further insight into the role of butyrylation in the process of adipogenesis, using small molecule compounds.

For this purpose, a series of derivatives of garcinol, a polyisoprenylated benzophenone isolated from Garcinia indica, were synthesized (11). These derivatives, unlike garcinol, were found to be non-toxic to the cells and showed a high specificity towards enzymatic activity inhibition of EP300. The derivatives were generated by internal cyclisation of garcinol with further addition of one or two functional groups to the remaining free $-\mathrm{OH}$ groups- giving rise to mono and disubstituted derivatives (Sup. fig. 2a, Fig. 3a panel i). Amongst these, a few monosubstituted derivatives could potently inhibit EP300 catalysed acetylation as well as butyrylation, while their disubstituted counterparts were unable to do so (Fig. 2a, panel i and ii, Sup. fig. 2b, panel i). This was probably due to increased steric hindrance brought about by the addition of an extra functional group that interfered with the interaction between the compounds and EP300.

An inverse correlation between catalytic rates of acylation reactions by EP300 and increasing acyl chain length had already been established previously (3). This led to our hypothesis that since butyrylation is catalysed by EP300 at a much slower rate compared to acetylation, one of the compounds which could not inhibit EP300 catalysed acetylation, might be able to inhibit the slower butyrylation reaction. In an in vitro butyrylation assay, using baculo virus expressed, full length recombinant human EP300 and bacterially expressed recombinant histone $\mathrm{H} 3$, we screened a set of garcinol derivatives. The results revealed a few potential candidates (Sup. fig. $2 b$, panel ii) as butyrylation inhibitors. Amongst them, LTK$14 \mathrm{~A}$, which is 13,14 dimethoxy isogarcinol (fig. 3A, panel i), was capable of inhibiting full length EP300 catalysed butyrylation at a concentration of $25 \mu \mathrm{M}$ with minimal effect on EP300 catalysed acetylation in a similar reaction system ( compare lane 6 in each of Fig. $2 \mathrm{~b}$ panels i and ii).

In order to understand the mechanism behind this selective inhibition by LTK-14A, we performed molecular docking analysis of LTK-14A and the EP300 lysine acetyltransferase catalytic domain. For this purpose, the crystal structure of LTK-14A was solved (Table 1, Sup. Fig.3d). LTK-14 (fig. 3a, panel i), a specific inhibitor of EP300 catalysed acetylation and butyrylation (Fig. 2a, panel I and ii) was taken for comparative docking onto the EP300 catalytic domain. The energy minimized analysis revealed that both LTK-14 and LTK-14A could potentially bind with the active site of EP300 catalytic domain forming several hydrophobic interactions (Fig.3a, panel ii). LTK-14, due to the presence of a free phenolic -OH group, could hydrogen bond with a carbonyl oxygen atom present in the peptide backbone of S1396 residue. However, LTK-14A has all of its phenolic -OH groups blocked and so it is incapable of forming such an interaction. This hydrogen bonding by LTK-14 could imply a stronger binding to the enzyme, while LTK$14 \mathrm{~A}$, by interacting with lesser number of residues presumably inhibits only the slower butyrylation reaction, although the interaction may not be strong enough for inhibiting the faster acetylation reaction.

Some of the residues with which LTK-14A could form hydrophobic interaction have already been reported before as being critical for targeting inhibition of enzymatic activity of EP300 (12). These residues include C1438, Y1446, Y1397 and W1436. To verify the molecular docking result, we generated a point mutant of the EP300 catalytic domain in which the mutation was introduced at one of the predicted positions C1438. This particular residue was chosen for downstream analysis as it was predicted to have the highest propensity for interaction with LTK-14A. The large Van der Waal's radius of sulfur in the side chain of cysteine residue might be responsible for the close proximity between the amino acid and the isoprenyl group of LTK-14A. Hence it was assumed that mutating the cysteine to alanine would reduce the proximity by curbing the length of the side chain at the $C \beta$ atom and thereby affect the interaction of LTK$14 \mathrm{~A}$ with the EP300 catalytic domain.

The C1438A catalytic domain was found to be equally active as the wild type KAT domain in an in vitro lysine acetyltransferase assay (data not shown). We parallely performed in vitro histone acylation assays using wild type and C1438A mutant of EP300 catalytic domain with different doses of LTK-14A. We observed that the wild type EP300 KAT domain catalysed butyrylation was inhibited by LTK-14A most prominently at $25 \mu \mathrm{M}$ concentration while acetylation was not inhibited (compare lane 6 in each of fig.3b panel i, ii, iii and iv). In contrast, neither acetylation nor butyrylation modification catalysed by the mutant EP300 catalytic domain could be inhibited by LTK-14A even at $25 \mu \mathrm{M}$ concentration (compare lane 6 in each of fig. 3c, panel i, ii, iii and iv). Taken together these data suggest that LTK-14A indeed interacts with $\mathrm{C} 1438$ within the active site of EP300 catalytic domain and this residue is critical for butyrylation reaction catalysis. 


\section{LTK-14A is a metabolically stable, cell permeable compound that significantly inhibits adipogenesis:}

Upon establishing that the semi-synthetic derivative of garcinol, LTK-14A is an EP300 acylation specific inhibitor in vitro, its cellular effects were tested using 3T3L1 cells. First we checked the cellular permeability and metabolic stability of the compound. It was found the LTK14A is highly cell-permeable and also metabolically stable as it could be detected by mass spectrometry of metabolites extracted from LTK-14A treated cells after 6 days (Fig. 4a).

MTT assay data showed that LTK-14A at $25 \mu \mathrm{M}$ concentration did not have any adverse effect on the cellular oxidoreductase activity, a signature of metabolic output of cells, (Fig. 4b) suggesting that LTK-14A is non-toxic to 3T3L1 even after 6 days. The effect of LTK-14A on the adipogenesis process was tested in the next step. The 3T3L1 cells were induced to undergo adipogenesis in the presence and absence of LTK-14A. Treatment with DMSO was taken as solvent control. The result shows that although there was minimal reduction in adipogenesis upon DMSO treatment, almost $60 \%$ inhibition in adipogenesis was observed in presence of LTK-14A as revealed by Oil Red-O staining, followed by spectrophotometric quantitation (Fig. 4c, panel i and ii).

The above data clearly establishes that although LTK-14A, is nontoxic to cells, it could inhibit the differentiation of the 3T3L1 cells. Presumably, LTK-14A treatment alters the gene expression essential for the process of adipogenesis, through its ability to inhibit butyrylation. Therefore, to understand the mechanism of LTK14A-mediated inhibition of adipogenesis, cells treated with LTK-14A were subjected to transcriptome analysis. The untreated and solvent treated cells were taken as control. The volcano plot (Fig. 5a, panel i,ii and iii) and heat map analysis (Fig. 5d) revealed that upon incubation with the compound, a large number of genes were found to be differentially expressed compared to DMSO treatment and untreated conditions.

In order to gain a better understanding of LTK-14A affected genes we focused on the common genes that were differentially expressed in the two cases- (1) LTK-14A versus no inhibitor and (2) LTK-14A versus DMSO. Amongst these common genes 2200 genes were found to be downregulated and 1309 genes were upregulated (Fig. 4d, panel i) with a majority of them to be protein coding genes (Fig. $4 \mathrm{~d}$, panel ii). A large proportion of these genes encoded for nucleic acid binding proteins (Fig. 5b) such as transcription factors (TFs). Interestingly, the major class of TFs affected were found to be zinc finger proteins (Sup. Fig. 4b).The gene ontology analysis revealed that LTK14A treatment predominantly affected the genes involved in lipid metabolism pathways (Fig. 5c, panel i and ii) which were minimally affected by DMSO alone (Fig. 5c, panel iii). The gene regulatory networks and the respective pathways were constructed with the major genes that were affected by LTK-14A treatment compared to DMSO treated and untreated conditions (Fig. 5e, Sup. Fig.4a). They collectively depict how LTK-14A, by inhibiting expression of a large number of genes that are interconnected via several pathways associated with lipid metabolism, brings about a collapse in the adipogenesis process as a whole.

A few of the pro-adipogenic genes downregulated by LTK-14A, as indicated by RNA sequencing, were selected for further validation (Fig. 5f). Quantitative PCR analysis corroborated that except for Cebpd, most of the other pro-adipogenic genes (Pparg, Cebpa, Adipoq, Lep, Slc2a4, Fasn and Fabp4) showed a marked downregulation upon LTK-14A treatment (Fig. 5g). Acss2, the gene encoding the enzyme speculated to generate butyryl CoA from butyrate, was also downregulated by LTK-14A (Sup. Fig.4c). The upregulation of anti-adipogenic genes was also verified by performing Q-PCR analysis for two such candidate genes- Kdm4c and Tead4 (Sup. Fig.4c). Collectively, the RNA-sequencing analysis along with the validation of gene targets further convincingly suggest that LTK-14A is capable of inhibiting the adipogenesis process in 3T3L1 cells, mainly by repressing the expression of important pro-adipogenic genes.

LTK-14A inhibits histone butyrylation in 3T3L1 cells, without affecting acetylation or other posttranslational modifications:

So far we have found that during adipogenesis, butyrylation of histones is enhanced. The natural compound garcinol derivative LTK-14A specifically inhibits EP300 mediated histone butyrylation in vitro and also adipogenesis in 3T3L1 cells. The RNA sequencing analysis, upon LTK-14A treatment revealed that it significantly inhibits the expression of pro-adipogenic genes. We were then interested to find out whether LTK-14A mediated downregulation of adipogenesis is causally linked to the inhibition of histone butyrylation. Histones were acid extracted from 3T3L1 cells under treated/untreated conditions with the 
compound and subjected to immunoblotting analysis. It was found that treatment with LTK-14A inhibited overall butyrylation of histones (Fig. 6a, panel $\mathrm{i}$ and ii). Among the specific modification sites tested, H3K23 and H4K5 butyrylation showed a significant decrease upon treatment with LTK-14A while butyrylation at other tested sites were affected minimally (data not shown). Acetylation of histones (H3K9, H3K18 and H4K12) was also found to be unaffected (Fig. 6b, panel i and ii) upon LTK-14A treatment. To establish the specificity of the LTK14A, the effect of LTK-14A on lysine and arginine methylation along with phosphorylation of histones were also investigated. We observed (Fig. 6c, panel $\mathrm{i}$ and ii) no significant changes in these marks upon the compound treatment, thereby indicating that in the 3T3L1 cells, LTK-14A specifically inhibits butyrylation.

\section{Inhibition of butyrylation reduces weight gain of high fat diet fed C57BL6/J mice:}

After observing the anti-adipogenic effect of LTK-14A on 3T3L1 cells, we investigated whether this compound could also affect adipogenesis at organismal level. For this purpose, C57BL6/J mice were maintained either on normal diet or on a high fat diet with or without LTK-14A. In due course of time, it was observed that the mice maintained on a high fat diet showed a significant increase in weight gain compared to those on chow diet, as expected. Interestingly, the mice maintained on a high fat diet along with LTK-14A had a weight gaining trend intermediate between the normal diet and high fat diet fed mice (Fig.7a, panel $\mathrm{i}$ and ii). Dissection of the mice revealed that the fat accumulation in high fat diet maintained mice was the highest, followed by those on high fat diet and LTK-14A, and then by those on normal diet (Fig. 7b, panel i). The epididymal fat pads (photographed with the testes as verification of tissue location but weighed after removal of testes) were separately weighed and the weights of the three groups showed the same trend as was seen for total body weights of the mice (Fig. 7b, panel ii and iii). The diet consumption pattern of the mice showed that the two groups on high fat diet with or without LTK$14 \mathrm{~A}$, had a similar consumption rate indicating that the reduced weight gain of the compound treated mice is not due to discrepancy in diet consumption (Table 2, Supplementary Information).

Hematoxylin and eosin staining of adipose tissue samples revealed morphology in high fat diet maintained mice typically carrying symptoms of onset of obesity i.e. the adipocytes were enlarged owing to lipid accumulation (Fig. 7c, panel i). The morphology of the tissues obtained from normal diet fed mice and the mice fed with compound treated high fat diet were closely similar to one another, as they both exhibited smaller adipocyte size. Along similar lines, the liver tissue of mice maintained on high fat diet showed symptoms of hepatic steatosis while the liver tissues of mice on normal diet and compound treated high fat diet did not exhibit any such features (Fig. 7c, panel ii). Taken together these data indicate that LTK-14A could inhibit adipogenesis even in the in vivo context, and thereby the development of obesity.

As the weight gain of mice on high fat diet was arrested by LTK-14A administration, we looked into the histone modification pattern in the liver as an indicator of the effect of LTK14A on possible alterations of histone post-translational modifications, especially butyrylation. Immunofluorescence staining of the liver tissue samples revealed that there was a significant increase in H4K5 butyrylation levels in high fat diet fed mice compared to normal chow diet fed mice in which the modification was barely detectable and it was almost completely inhibited upon LTK-14A treatment (Fig. 7d, panel iii and vi). On the other hand, no significant difference in H4K12 (Fig. 7d, panel i and iv) and H3K14 acetylation (Fig. 7d, panel ii and v) levels in these liver samples of normal diet and high fat diet fed mice could be observed. The enhanced level of butyrylation upon high fat diet consumption could allude to the importance of this modification in the context of adipogenesis and obesity, further corroborated by the finding that H4K5 butyrylation could be almost completely inhibited by treatment with LTK-14A (Fig. 7d, panel iii and vi).

Furthermore, histone modification pattern in the adipose tissues was studied with acid extracted histones from the epididymal fat pads. H4K5 butyrylation was reduced in the LTK-14A treated samples compared to those of high fat diet fed mice (Fig. 7e, panel i and iii). H3K14 acetylation levels were relatively unchanged across all the three different groups (Fig. 7e, panel i and ii).

The bioavailability of LTK-14A in mice was verified using the liver extracts from the compound treated animals by LC-MS analysis. The results show that indeed LTK-14A could be detected in the liver extracts of all the compound treated mice as shown in the ESI spectra of the analytes (Fig. 7f).

The butyrylation specific inhibitor arrests as well as reduces weight of genetically obese $\mathrm{db} / \mathrm{db}$ mice: 
The attenuation of weight gain in C57BL6/J mice had been achieved with LTK-14A being added within the high fat diet from the very initiation point of the experiment. We then proceeded with a different approach to test whether LTK-14A could also prevent weight gain in mice that were already obese. Genetically obese $\mathrm{db} / \mathrm{db}$ mice were taken as the model system for this purpose. Heterozygous mutants $\mathrm{db}+/$ - mice were taken as control for the homozygous mutant $\mathrm{db} / \mathrm{db}$ mice. These hyperphagic $\mathrm{db} / \mathrm{db}$ mice were orally administered with LTK-14A daily at the same dosage as before (50 mg/Kg body weight). We observed that upon oral gavaging of LTK-14A, the overweight mice not only showed an arrest in weight gain, but their body weights were significantly reduced by the end of one month of treatment (Fig. 8a panel $\mathrm{i}$, ii and iii). The food intake of LTK-14A treated mice was marginally less than that of the vehicle treated mice (Table 2, Supplementary Information) while both groups had significantly greater energy intake compared to the $\mathrm{db}+/$ - mice. Average weight of the liver (Fig. 8b, panel $\mathrm{i}$ and ii) and epididymal fat pads (Fig. 8c, panel $\mathrm{i}$ and ii) was the highest for $\mathrm{db} / \mathrm{db}$ mice given vehicle control and least for the $\mathrm{db}+/$ mice, while that for the compound treated $\mathrm{db} / \mathrm{db}$ mice was intermediate. In vivo Echo MRI of live mice revealed that total adiposity i.e. fat mass of the mice was significantly lowered upon LTK-14A treatment (Fig. 8d, panel i) while lean mass was marginally affected (Fig. 8d, panel ii). The negligible reduction in lean mass could be due to lower feed intake of the compound treated mice that led to the lowering of both fat mass and muscle mass. However, LTK-14A treatment showed significant effect on lipid accumulation compared to muscle mass development. Hematoxylin and eosin staining of liver tissues indicated a high degree of hepatocyte ballooning in $\mathrm{db} / \mathrm{db}$ mice given vehicle control, a signature of high inflammatory response due to excessive lipid accumulation and peroxidation (Fig. 8e, panel i). Such features were absent in the liver tissues of $\mathrm{db}+/-$ mice and $\mathrm{db} / \mathrm{db}$ mice treated with LTK-14A. Moreover, hematoxylin and eosin staining of the adipose tissues showed greatest degree of adipocyte hypertrophy for the vehicle treated $\mathrm{db} / \mathrm{db}$ mice, followed by compound treated $\mathrm{db} / \mathrm{db}$ mice and $\mathrm{db}+/$ - mice in decreasing order (Fig. $8 \mathrm{e}$, panel ii). Similar to the high fat diet induced obesity model, the more obese homozygous leptin receptor mutant mice $(\mathrm{db} / \mathrm{db})$ liver had greater level of $\mathrm{H} 4 \mathrm{~K} 5$ butyrylation compared to leaner heterozygous mutant mice ( $\mathrm{db}+/-)$ (Fig $8 f$ panel iii and vi). Moreover, LTK-14A treated $\mathrm{db} / \mathrm{db}$ mice liver showed a decrease in H4K5 butyrylation compared to vehicle control treated mice liver. In contrast, H4K12 (Fig. 8f panel $\mathrm{i}$ and iv) and H3K14 acetylation (Fig. 8f panel ii and v) were only partially upregulated in the $\mathrm{db} / \mathrm{db}$ mice and did not get significantly reduced upon LTK-14A treatment.

Immunoblotting with acid extracted histones from epididymal fat pads showed that H4K5 butyrylation was reduced in the adipose tissues of LTK-14A treated mice (Fig. $8 \mathrm{~g}$, panel $\mathrm{i}$ and iii) compared to vehicle control treated obese mice. H3K14 acetylation was not significantly altered in any of the three groups (Fig. 8g, panel i and ii).

Collectively these data establish that obesity can not only be prevented but also reverted by specific inhibition of H4K5 butyrylation, thereby highlighting the importance of this modification in the context of adipogenesis.

\section{Discussion}

Reports on the physiological relevance of acylation modifications are scanty. Breakthroughs have been made in establishing the importance of butyrylation in spermatogenesis (8), $\beta$-hydroxybutyrylation in starvation response (13) and crotonylation in inflammatory response, digestive system, cancer and HIV latency $(10,14,15,16)$. Lysine propionylation has also been reported as an activation mark on histones that could be useful in various physiological processes (17). Mass spectrometry based global acylome has been reported in several model organisms- starting from human pathogen Pseudomonas aeruginosa (18) to eukaryotes like papaya (19), rice plants $(20,21)$ and zebrafish (22). The list of these modifications seems to be increasing continuously, with the recent additions of histone serotonylation (23), lactylation (24) and dopaminylation (25). The fact that serotonin and dopamine are chemical neurotransmitters while lactate is derived from pyrvate, a glycolytic end product, and all these molecules efficiently modify histones, thereby mediating gene expression in several tissues, bear testimony to the hypothesis that the abundance levels of cellular metabolites in different physiological contexts can lead to modification of histones with a plethora of diverse chemical moieties that can selectively modulate gene expression patterns.

During adipogenesis, due to a surge in fatty acid synthesis, an increased abundance of butyrate could lead to butyrylation of histones to keep the highly transcribed genes transcriptionally active. Despite the 
redundancy in its functionality with histone acetylation, the actual importance of butyrylation could only be shown using a pharmacological approach. LTK-14A, a semi-synthetic small molecule modulator was able to selectively inhibit p300 catalysed butyrylation without affecting acetylation in vitro. This very property of the compound was explored to establish the significance of butyrylation independent of acetylation in adipogenesis whereby the compound administration inhibited adipogenesis through repression of proadipogenic gene expression. However amongst these genes CEBPd did not seem to be affected by LTK$14 \mathrm{~A}$ possibly because the expression of this gene is less prone to be regulated by histone butyrylation in its promoter. Acss2, which encodes the enzyme responsible for butyryl CoA biosynthesis was also downregulated which might have further contributed to reduced butyrylation. Amongst the different histone modification patterns investigated, predominantly $\mathrm{H} 4 \mathrm{~K} 5$ butyrylation was consistently affected by LTK-14A in different cell line and mice models, while the other site specific butyrylation marks were relatively unaltered indicating that there may be a hierarchy in the importance of these modifications in regulation of adipogenesis. Global histone acetylation levels in liver were previously reported to remain nearly unchanged even after high fat diet consumption (26). In the present study we have found that both diet induced and genetic models of obesity did not show any drastic variation in acetylation at the global level. Under normal circumstances acetyl CoA is present in high abundance. Thus even if there were fluctuations in acetyl CoA levels, EP300 acetyltransferase activity was not affected by limitation of cofactor availability. On the other hand, butyryl CoA being a rare acyl CoA species, its basal abundance would be much less than its corresponding Km (Michaelis Menten constant) for EP300, which could enable the enzyme to detect small changes in butyryl CoA concentrations. Possibly, high fat diet consumption or excessive dietary intake of fuel led to an increase in butyryl CoA levels which were reflected in an enhanced H4K5 butyrylation in the liver samples of obese mice. Upon high fat diet consumption, acetylation on histones was affected to a different extent at various sites; either remaining unchanged or minimally reduced (26). As reported before, H3K14 acetylation did not get altered much in obese mice adipose tissues compared to lean mice. However, unlike in the case of liver and cell line model of adipogenesis, we did not observe any enhanced H4K5 butyrylation in fat pads of obese mice. A possible explanation for this could be that all animal experiments were performed in adult mice in which the adipose tissues were already developed. Inside these tissues, more than $2 / 3 \mathrm{rd}$ of the cell subpopulation is composed of adipocytes, contributing 80 percent of the tissue volume (27). Probably due to predominance of adipocytes, no global increase in H4K5 butyrylation could be seen in the hypertrophied adipose tissues of obese mice.

In the 21st century, obesity has become a major epidemic leading to several other clinical disorders such as type 2 diabetes, arteriosclerosis and hypertension. It is associated with increased adipose tissue mass i.e. increased adipocyte number (hyperplasia) or size (hypertrophy) due to dysregulation in adipogenesis. Histone acetylation inhibition could be regarded as a possible method for attenuating adipogenesis. But acetylation is a widespread phenomenon that is required in several other physiological processes besides adipogenesis. Hence, treatment with an acetyltransferase inhibitor at a particular range of concentration could have undesirable side effects and toxicity. In contrast, histone butyrylation is a rare event because the physiological levels of butyryl CoA are much lower than that of acetyl CoA and increase during adipogenesis. The increased importance of histone butyrylation in adipogenesis makes it an attractive target for chemical based inhibition of adipogenesis and thereby controlling obesity (Graphical abstract). In this aspect, additional improvements can be made in the designing of LTK-14A structure to increase its efficacy for butyrylation inhibition without losing its unique specificity.

\section{Materials and Methods}

Animals and treatment: Male C57BL/6J mice (8 weeks old) were bred in house and acclimatized for 1 week prior to experiments. Mice were maintained on a 12 hour light/dark cycle and given ad libitum access to food and water. Experiments were carried out in accordance with the Committee for the Purpose of Control and Supervision of Experiments on Animals (CPCSEA) guidelines and were approved by the Institutional Animal Ethics Committee of JNCASR (201/GO/Re/S/2000/CPCSEA dated 1st June, 2000 Renewed in Aug 2015). The age-matched animals were maintained on a standard laboratory chow diet (AMRUT M/s Krishna Valley Agrotech, Maharashtra) or high fat diet (60 kcal\% fat) (Research Diets, Cat. No. D12492) with or without LTK-14A (50 mg/Kg body weight) and water. LTK-14A was mixed with 
the diet according to dosage requirements by the researchers. Body weight and food intake was measured twice every week.

For the experiment on genetically obese mice, male $\mathrm{db} / \mathrm{db}$ mice and $\mathrm{db}+/-$ mice (8-10 weeks old) bred in house were obtained and acclimatized for 1 week prior to experiments. Mice were maintained on a 12 hour light/dark cycle at $22+/-3$ oC and a relative humidity of $55 \%$ and given ad libitum access to food and water. The experiments were carried out in accordance with the Committee for the Purpose of Control and Supervision of Experiments on Animals (CPCSEA) guidelines, Government of India and was approved by the Institutional Animal Ethics Committee of CSIR-CDRI, Lucknow (IAEC/2020/115/Renew0/SI.No.09). The age-matched animals were maintained on a standard laboratory pelleted diet (Altromin International, Cat. No. 1320) and water. $\mathrm{db} / \mathrm{db}$ mice were oral gavaged $(50 \mathrm{mg} / \mathrm{kg}$ ) with LTK14A in $0.5 \%$ carboxymethyl cellulose, equal volume of the vehicle was orally gavaged to control $\mathrm{db} / \mathrm{db}$ mice for a total period of 30 days. $\mathrm{db}+/$ - animals were kept as untreated normal control group. The body weights and feed intake was recorded every week throughout the experimental period. The lean and fat mass of the mice were determined using Echo MRI in live mice on day 15th and day 30th.

Cell culture: The mammalian cell line 3T3L1 was obtained from ATCC (American Type Culture Collection) and was grown in DMEM (Dulbecco's Modified Eagle's Medium). The growth medium was supplemented with $10 \%$ FBS (Fetal Bovine Serum). The cell line was grown in $37^{\circ} \mathrm{C}$ incubator with $5 \%$ $\mathrm{CO} 2$ and $90 \%$ relative humidity.

Adipogenesis and Oil Red-O staining: 3T3L1 preadipocytes were grown to confluency and 24 hours post-confluence, cells were incubated with differentiation medium (growth medium containing $1 \mu \mathrm{M}$ Dexamethasone, $0.5 \mathrm{mM} \mathrm{IBMX}$ and $1 \mu \mathrm{g} / \mathrm{ml}$ insulin) for 2 days in the presence/absence of LTK-14A. Then the cells were supplemented with maintenance medium with/without LTK-14A (growth medium with $1 \mathrm{\mu g} / \mathrm{ml}$ insulin only) every alternate day. On the 6th day post induction of differentiation the cells were fixed with $3.7 \%$ formaldehyde solution followed by staining with Oil Red-O dye (Sigma-Aldrich) (prepared in $60 \%$ isopropanol) for 1 hour at room temperature. The incorporated dye was extracted using isopropanol and absorbance values were determined spectrophotometrically at $510 \mathrm{~nm}$ to estimate total amount of lipid accumulation under different experimental conditions.

Acid extraction of histones and immunoblotting: 3T3L1 cells were washed with ice-cold PBS and then resuspended in Triton extraction buffer (PBS containing 0.5\% Triton X-100 (v/v) and $2 \mathrm{mM} \mathrm{PMSF).}$ The cells were allowed to lyse on ice for 10 minutes, spun down, washed with PBS and the process was repeated another time. Then the proteins were acid extracted with $0.2 \mathrm{~N} \mathrm{HCl}$ for two hours on ice. The debris was spun down and then histones were precipitated by incubating the supernatant with $33 \%$ trichloroacetic acid (Sigma-Aldrich) at $4 \square \mathrm{C}$ for 30 minutes. The histones were then pelleted down and then given two washes with acetone. The pellet was allowed to air-dry for 10 minutes and then resuspended with $50 \mathrm{mM}$ Tris- $\mathrm{Cl}, \mathrm{pH}$ 7.4. Extraction of histones from epididymal fat pads was performed by mechanically homogenizing the adipose tissues in Triton extraction buffer and then following the same subsequent steps as mentioned before, except for the step of acid extraction with $\mathrm{HCl}$, in which the incubation was performed for four hours on ice.

The samples were then run in 15\% SDS-PAGE, transferred onto PVDF membrane (Milipore) in transfer buffer (25 mM Tris, $192 \mathrm{mM}$ glycine, $0.036 \%$ SDS in 20\% methanol), blocked with $5 \%$ skimmed milk solution to prevent non-specific antibody binding and finally probed with antibodies against acetylation and butyrylation marks prepared in $1 \%$ skimmed milk or FBS solution ( $5 \%$ BSA, $1 x$ TBS, $0.1 \%$ Tween20). HRP-conjugated secondary antibodies (Abcam) were used against the primary antibodies to obtain a chemiluminiscent signal in the presence of SuperSignal West Pico Chemiluminiscent Substrate (Thermo Scientific) which was developed using the VersaDoc imaging system (Biorad).

Synthesis of LTK14A: Isolation of garcinol from Garcinia indica and synthesis of isogarcinol from it were carried out following the protocols as mentioned in Mantelingu et al, (2007) Chem Biol (11). For LTK-14A synthesis, anhydrous potassium carbonate $(0.41 \mathrm{mmol})$ was added to a stirred solution of isogarcinol (100 $\mathrm{mg}, 0.16 \mathrm{mmol})$ in dry acetone. The mixture was stirred for 1 hour at room temperature followed by dropwise addition of dimethyl sulfate $(0.41 \mathrm{mmol})$ under nitrogen gas atmosphere. The resulting reaction mixture was further stirred at room temperature for 14 hours. Progress of the reaction was monitored through thin layer chromatography. Acetone was evaporated from the reaction mass, ice was added and 
acidified with $5 \%$ aqueous $\mathrm{HCl}(\mathrm{pH} \sim 5-6)$. A pale brown precipitate was observed, which was filtered and washed with cold water. The dry mass was then chromatographed on silica gel using $5-6 \%$ ethyl acetate in hexane as eluting solvent mixture. $720 \mathrm{mg}(69 \%)$ LTK14A was isolated as white solid. NMR spectral analysis was consistent with the desired product (Sup. Fig. 2A). A $20 \mathrm{mg}$ sample was dissolved in hot methanol and placed in a flat surface glass vial with access to very slow solvent evaporation. Crystals as colorless needles were isolated. The purity of the synthesized LTK-14A was found to be more than $98 \%$ as verified by HPLC (Sup. Fig. 2C) and only the protonated species of LTK-14A were observed in the ESI spectra of the corresponding fraction (Sup. Fig. 2B).

X-ray crystal structure determination for LTK14A (CCDC: 1969185): Suitable single crystal with approximate dimensions of $0.35 \times 0.18 \times 0.15 \mathrm{~mm} 3$ was used for X-ray diffraction analyses by mounting on the tip of a glass fibre in air. Data were collected on a Bruker kappa apex 2 with Mo Ka $(\lambda=0.71073 \AA)$ at 296 (2) K. The structure was solved by direct method using program SHELXL-97 and subsequent Fast Fourier Transform technique. Crystallographic data and experimental details for LTK14A are summarized in Table 1 and Sup. Fig. 3D.

Targeted analysis of butyryl CoA and untargeted analysis of intracellular metabolites by Ultraperformance liquid chromatography coupled with time-of-flight mass spectrometer (Q-TOF LC/MS):Profiling of intracellular metabolites as well as targeted analyses of butyryl CoA was performed on an agilent 1290 Infinity LC system coupled to Agilent 6545 Accurate-Mass Quadrupole Time-of- Flight (QTOF) with Agilent Jet Stream Thermal Gradient Technology. The UPLC system was assembled with a Diode array detector (DAD) and autosampler. The Chromatographic separation was achieved on Agilent ZORBAX SB- C18 column $(2.1 \times 100 \mathrm{~mm}, 1.8 \mu \mathrm{m})$ as stationary phase. For untargeted analysis, the mobile phase consisted of a linear gradient of $100 \mathrm{mM}$ ammonium formate $(A)$ and Acetonitrile (B): 0-10.0 $\min , 30-80 \%$ B (v/v); 10.0-15.0 min, 80-100\% B (v/v); 15.0-20.0 min, 100\% B (v/v); 20.0-21.0 min, 100$30 \% \mathrm{~B}(\mathrm{v} / \mathrm{v}) ; 21.0-25.0 \mathrm{~min}, 30 \% \mathrm{~B}$. For targeted analysis, the mobile phase consisted of a linear gradient of $100 \mathrm{mM}$ ammonium formate $(A)$ and Acetonitrile (B): 0-5.0 min, 0-50\% B (v/v); 5.0-6.5 min, 50-100\% B $(\mathrm{v} / \mathrm{v}) ; 6.5-8.0 \mathrm{~min}, 100 \%$ B (v/v); 8.0-9.0 min, 100-0\% B (v/v); 9.0-15.0 min, 100\% A. The sample was dissolved in $1 \mathrm{~mL}$ methanol (LCMS Grade), centrifuged and supernatant was taken for UPLC-QTOF-MS analysis. The column was reconditioned for 5 minutes prior to the next injection. The flow rate was 0.5 $\mathrm{mL} / \mathrm{min}$, and the injected volume was $20 \mu \mathrm{L}$. The UPLC was connected to the MS analysis was performed on an Agilent 6545 Accurate-Mass Q-TOF/MS system with an electrospray ionization (ESI) source. Considering the MS conditions, positive ion mode was used to obtain high-resolution mass spectra. The ESI source parameters were: drying gas (N2) flow, $8 \mathrm{~L} / \mathrm{min}$; drying gas temperature, $200^{\circ} \mathrm{C}$. Other parameters were set as nebuliser gas, 35 psig; capillary voltage, $3000 \mathrm{~V}$; skimmer voltage, $65 \mathrm{~V}$; nozzle voltage $1000 \mathrm{~V}$ and fragmentor voltage $150 \mathrm{~V}$. The data acquisition on the LC-QTOF was performed using Agilent MassHunter Acquisitionsoftware (Agilent Technologies, Santa Clara, CA, USA). The data were deconvoluted into individual chemical peaks with Agilent MassHunter Qualitative Analysis (MassHunterQual, Agilent Technologies). For comparison of butyryl CoA levels under different conditions, the peak area of butyryl CoA fraction was normalised to that of all other metabolites and this area sum percent was used as a measure of the abundance level of butyryl CoA.

Site directed mutagenesis: Point mutation was introduced into the p300 catalytic domain sequence cloned within pET-28b plasmid by using the Quikchange site directed mutagenesis kit (Agilent). Briefly, the template was mixed with Pfuturbo DNA polymerase, designed forward and reverse primers and dNTPs for PCR (Initial denaturation for 30 seconds at $95^{\circ} \mathrm{C}$; Cycles: $95^{\circ} \mathrm{C} 30^{\prime \prime}, 55^{\circ} \mathrm{C}$ for $60^{\prime \prime}, 68^{\circ} \mathrm{C}$ for $7^{\prime}$; final elongation at $68^{\circ} \mathrm{C}$ for $5^{\prime}$.) The mixture was then incubated with Dpnl enzyme for 2.5 hours for removal of the parent wild type sequence followed by transformation of competent XL10Gold cells and kanamycin selection of positive transformants. The inserted mutation was verified by sequencing and alignment with the wild type sequence using ClustalOmega software.

Purification of recombinant full-length p300 and p300 catalytic domain: Polyhistidine tagged recombinant p300 was purified from Sf21 insect cells by Ni-NTA based affinity chromatography. These cells were infected by the baculovirus containing p300 for 72 hours. Once the cells showed signs of infection, they were scraped off and centrifuged to separate the spent media. The cell pellets were resuspended in ice cold homogenisation buffer (20 mM Tris, pH 7.5; $20 \mathrm{mM}$ imidazole, 10\% glycerol, 0.2 
mM EDTA, $300 \mathrm{mM} \mathrm{KCl,} \mathrm{0.1 \% NP-40,} 2 \mathrm{mM}$ PMSF, $2 \mathrm{mM}$ ßmercaptoethanol) and then homogenised using a Dounce homogeniser ( 5 cycles, 6 strokes per cycle with 5 minutes interval between each cycle). The cell debris was separated by centrifugation and then the lysate was incubated with pre-equilibriated Ni-NTA slurry for 2.5 hours at $4^{\circ} \mathrm{C}$ on an end-to-end rotor. Then the beads were washed with wash buffer (20 mM Tris, pH 7.5; 40 mM imidazole) nine times to remove unwanted contaminants. Finally p300 was eluted using elution buffer (20 mM Tris, pH 7.5; $250 \mathrm{mM}$ imidazole).

Polyhistidine tagged p300 catalytic domain cloned in pET28b plasmid and Sirt2 were used for cotransforming BL21(DE3) cells. At first, $50 \mathrm{ml}$ primary culture was prepared in Luria broth containing kanamycin $(50 \mu \mathrm{g} / \mathrm{ml})$ and ampicillin $(100 \mu \mathrm{g} / \mathrm{ml})$ (HIMEDIA) and then it was scaled up 10 times to prepare secondary culture. Incubation was done at $37 \square \mathrm{C}, 180 \mathrm{rpm}$ till O.D. reached 0.37 followed by induction with $0.2 \mathrm{mM} \mathrm{IPTG}$ and a further incubation at $30 \square \mathrm{C}$ for 4 hours. Cells were pelleted at 6000 rpm, $4 \square \mathrm{C}$ for 10 minutes and stored at $-80 \square \mathrm{C}$. Resuspension and homogenisation of the pellet was carried out in homogenisation buffer (20 mM Tris, $\mathrm{pH} 7.5 ; 20 \mathrm{mM}$ imidazole, $10 \%$ glycerol, $0.2 \mathrm{mM}$ EDTA, $300 \mathrm{mM} \mathrm{KCl}, 0.1 \% \mathrm{NP}-40,2 \mathrm{mM}$ PMSF, $2 \mathrm{mM}$ ßmercaptoethanol). 3 cycles of sonication were done for 3 minutes at $35 \%$ amplitude with 5 minutes interval between each cycle. The cell debris were separated by pelleting at $12000 \mathrm{rpm}$ for 30 minutes at $4 \square \mathrm{C}$. The supernatant was equilibriated with Ni-NTA beads slurry (Novagen) in an end-to-end rotor for 3 hours at $4 \mathrm{C}$. Eight washes were given with wash buffer ( 20 $\mathrm{mM}$ Tris, $\mathrm{pH} 7.5 ; 40 \mathrm{mM}$ imidazole). Elution was carried out in batches with elution buffer $(20 \mathrm{mM}$ Tris, $\mathrm{pH}$ 7.5; $250 \mathrm{mM}$ imidazole).

The enzymatic activity of the proteins was estimated by filter binding assay.

In vitro filter binding assay: Different dilutions of purified enzyme were incubated in reaction buffer (50 $\mathrm{mM}$ Tris-HCl pH 7.5, $1 \mathrm{mM}$ DTT, $10 \%$ Glycerol, $2 \mathrm{mM} \mathrm{PMSF}$ ) with $10 \mathrm{mM} \mathrm{NaBu}$, tritiated acetyl CoA (50 $\mu \mathrm{Ci}$ ) (PerkinElmer, Part No: NET290050UC) and Xenopus histone H3 (500 ng) for 30 minutes at 30 $\square \mathrm{C}$. The reaction mixture was then spotted on P81 filter paper and the paper was allowed to dry at room temperature. The paper was washed in wash buffer $(50 \mathrm{mM}$ sodium bicarbonate and $50 \mathrm{mM}$ sodium carbonate), dried at $90 \square \mathrm{C}$ and immersed in enhancing solution (2.5\% PPO and $0.25 \%$ POPOP in toluene) for taking radioactive counts using liquid scintillation counter (Perkin Elmer, MicroBeta2 system).

In vitro acylation (acetylation/butyrylation) assay: Acylation reactions were performed in reaction buffer (25 mM Tris- $\mathrm{HCl}$ pH 7.5, $100 \mathrm{mM} \mathrm{NaCl}, 0.1 \mathrm{mM}$ EDTA, $1 \mathrm{mM}$ DTT, $10 \%$ Glycerol, 1x PIC) with $100 \mathrm{ng} / \mathrm{mL}$ TSA, and $50 \mu \mathrm{M}$ Butyryl-CoA/Acetyl CoA. Xenopus histone $\mathrm{H} 3(1 \mu \mathrm{g})$ was used as the substrate and full length p300 or p300 catalytic domain $(10,000 \mathrm{cpm}$ activity) was used as the enzyme. Reactions were incubated in presence or absence of butyrylation inhibitor for 10 minutes at $30{ }^{\circ} \mathrm{C}$, followed by initiation of the acetylation/butyrylation reaction by the addition of acetyl CoA/butyryl CoA. After a further 10 minutes, reactions were stopped by addition of Laemmli buffer and samples were used for immunoblotting.

Molecular docking analysis: Glide module of Schrodinger suite was used to dock LTK-14 and LTK-14A to p300 catalytic domain (PDB: 3BIY). Chimera was used as visualizing software. The protein was prepared using the wizard tool in Maestro version 10.2. The crystal structure of LTK-14A (CCDC 1969185) and LTK-14 (CCDC 645420) were used as the most optimized and energy minimized ligand structures. The receptor grid for p300 catalytic domain was generated by specifying the binding (active) site residues using Receptor grid generation in the Schrodinger Glide application. Once the receptor grid was generated, the ligands LTK-14 and LTK-14A were docked to the p300 catalytic domain using Glide version 5.8 molecular docking.

MTT assay: 12500 cells $/ \mathrm{ml}$ were seeded in presence/absence of LTK-14A in 96-well plate and at different intervals of time ( 2 days, 4 days, 6 days) , $1 / 10$ th volume of $5 \mathrm{mg} / \mathrm{ml} \mathrm{MTT}$ solution was added to it followed by incubation at $37^{\circ} \mathrm{C}$ for 3 hours. The formed crystals were dissolved in DMSO and spectrometric estimation of colour intensity was done at $570 \mathrm{~nm}$.

Qualitative real time PCR: Total RNA was isolated from the 3T3L1 cells by phenol-chloroform method. In case of LTK-14A treatment, 3T3L1 cells were allowed to differentiate for 7 days following the standard adipogenesis protocol, in presence of the compound at $25 \mu \mathrm{M}$ concentration. The control experiments were 3T3L1 cells differentiated with or without DMSO as solvent control and differentiation was done only 
with the addition of only the cocktail of chemicals for adipogenesis. RNA was isolated from the cells after the period of 7 days in all three cases. The isolated RNA samples were treated with 2 units/ $\mu \mathrm{L}$ DNase I to remove any residual DNA followed by overnight ethanol precipitation at $-80 \square \mathrm{C}$. RNA integrity was checked in $1 \%$ agarose gel. $2 \mu \mathrm{g}$ RNA was used for cDNA synthesis using MMLV reverse transcriptase (Sigma) in presence of $0.5 \mathrm{mM}$ dNTP and $3.5 \mu \mathrm{M}$ oligo dT (Sigma). QRT-PCR amplification was done in kappa SYBR green reagent (Biosystems) (Initial denaturation for 10 minutes at $95^{\circ} \mathrm{C}$; Cycles: $95^{\circ} \mathrm{C} 15^{\prime \prime}$, $65^{\circ} \mathrm{C}$ for $30^{\prime \prime}, 72^{\circ} \mathrm{C}$ for $30^{\prime \prime}$ ) with the help of Real time PCR instrument (Applied Biosystems). For estimation of relative fold change in RNA expression, the following calculation was done: Relative fold change $=2^{-\Delta \Delta \mathrm{Ct}}$, where $\Delta \Delta \mathrm{Ct}=\Delta \mathrm{Ct}$ value of sample $-\Delta \mathrm{Ct}$ value of control and $\Delta \mathrm{Ct}=\mathrm{Ct}$ value of target $-\mathrm{Ct}$ value of internal control eg. actin for individual sample. The sequences of primers are provided in Table 7.

Transcriptomics analysis by RNA-seq: RNA integrity was checked by Agilent Bioanalyzer 2100, only samples with clean rRNA peaks were used. Libraries for RNA-seq were prepared according to KAPA Stranded RNA-Seq Kit with RiboErase (KAPA Biosystems, Wilmington, MA) system. Final library quality and quantity were analyzed by Agilent Bioanalyzer 2100 and Life Technologies Qubit3.0 Fluorometer, respectively. 150 bp Paired-end sequencing was performed on Illumina HiSeq 4000 (Illumnia Inc., San Diego, CA).

House mouse (Mus musculus, strain C57BL/6J) genome (mm10) was downloaded from GENCODE and indexed using Bowtie2-build with default parameters. Adapter removal was done using Trim Galore $(\mathrm{v}$ 0.4.4) and each of the raw Fastq files were passed through a quality check using FastQC. PCR duplicates were removed using the Samtools 1.3.1 with the help of 'rmdup' option. Each of the raw files was then aligned to mm10 genome assembly using TopHat2 with default parameters for paired-end sequencing as described in (28). After aligning, quantification of transcripts was performed using Cufflinks and then Cuffmerge was used to create merged transcriptome annotation. Finally differentially expressed (DE) genes were identified using Cuffdiff. The threshold for DE genes was log2 (fold change) $>1.5$ for up regulated genes and log2 (fold change) $<1.5$ for down regulated genes with $p$-value $<0.05$.

GO enrichment analysis

Gene Ontology (GO) analysis was performed in PANTHER (29). Significant enrichment test was performed with the set of differentially expressed genes in PANTHER and Bonferroni correction method was applied to get the best result of significantly enriched biological processes.

Fisher's exact test

Fisher's exact test was performed in PANTHER Gene Ontology (GO) where p-value significance was calculated based on the ratio of obtained number of genes to the expected number of genes $(O / E)$ considering the total number of genes for the respective pathway in Mus musculus with a FDR of $<0.05$.

Heatmap and clustering of genes

Unsupervised hierarchical clustering method was performed using Cluster 3.0 (30) with Pearson Correlation and average linkage rule. Gene expression data (FPKM of all samples) was taken and log2 transformed. Low expressed $(F P K M<0.05)$ and invariant genes were removed. Then genes were centered and clustering was performed based on differential expression pattern of genes and fold change. Finally, the heatmap was visualized in Java TreeView 3.0.

Biological analysis of differentially expressed transcripts and pathway regulatory network modeling Statistically significant differentially expressed transcripts were subjected to GO and Pathway enrichment using DAVID tool. Only those GO and pathways with a FDR score of $<=0.05$ was considered for further downstream analysis. Key biologically dysregulated GO and Pathways along with the differentially expressed genes was provided as an input to Pathreg algorithm from Theomics International Pvt Ltd, Bangalore, India for gene regulatory network modeling. The result (nodes and edges) of the Pathreg algorithm was provided as an input to Cytoscape v2.8.2 to identify key nodes and edges that could be representative of the gene regulatory changes upon treatment.

Identification of pro-adipogenic and anti-adipogenic factors was performed by manual curation of candidate genes from previously published reports and Mouse Genome Informatics international database of genes involved in fat cell differentiation.

Hematoxylin and eosin staining of liver and adipose tissues: Staining was performed on paraffin embedded sections of liver and epididymal fat pads having the thickness of $5 \mu \mathrm{m}$ and $10 \mu \mathrm{m}$ respectively. Briefly, the sections were deparaffinized in xylene, followed by immersion in absolute alcohol, $90 \%$ ethanol, $70 \%$ ethanol, $50 \%$ ethanol and water before staining with hematoxylin for 5-10 minutes. The 
sections were immersed in above mentioned solutions in reverse order before counter-staining in eosin for 15 seconds. Then the samples were dipped in absolute ethanol and xylene before mounting with DPX.

Immunohistochemistry: Collected liver tissue samples were stored in 4\% paraformaldehyde for 24 hours after which they were cryoprotected in 30\% sucrose solution for two weeks. Cryosections were performed at $7 \mu \mathrm{m}$ sections using Cryostat LeicaCM1850 UV. For staining, the tissue sections were washed with PBS followed by antigen retrieval with $0.01 \mathrm{M}$ citrate buffer $(\mathrm{pH} 6)$. The tissues were then permeabilised with $0.3 \%$ Triton X-100/PBS (PBST) and blocked with $2 \%$ serum followed by incubation with primary antibodies overnight at $4 \square \mathrm{C}$. The next day, secondary antibody incubation was carried out for one hour at room temperature followed by staining of the nuclei with Hoechst 33342 and mounting with $70 \%$ glycerol. Images were acquired by confocal microscopy. For quantitation, intensity of each modification specific staining was normalized with respect to the Hoechst staining intensity.

Reagents and antibodies: The media used for cell culture was purchased from Sigma-Aldrich; DMEM (Ref: 1152). Several other cell culture reagents were obtained from HIMEDIA, such as PBS (Ref: TL1006), Trypsin-EDTA solution 10X (Ref: TCL070) and Antibiotic antimyotic solution 100X (Ref: A002A). Multiple reagents used for cell culture were obtained from Sigma-Aldrich: Insulin (Ref: 16634), Dexamethasone (Ref: D4902), IBMX (Ref: 15879). TRIzol reagent was obtained from ambion life technologies (Ref: 15596018). The commercial antibodies used in this study are Pan anti-butyryllysine (PTM Biolabs, SKU: PTM 301), Butyryl-Histone H4 (Lys 12) rabbit pAb (PTM Biolabs, SKU: PTM 308), Butyryl-Histone H3 (Lys 9) rabbit pAb (PTM Biolabs, SKU: PTM 305), Butyryl-Histone H3 (Lys 23) mouse mAb (PTM Biolabs, SKU: PTM 307), Butyryl-Histone H4 (Lys 8) rabbit pAb (PTM Biolabs, SKU: PTM 311), Butyryl-Histone H4 (Lys 5) rabbit pAb (PTM Biolabs, SKU: PTM 313), Butyryl-Histone H3 (Lys 27) rabbit pAb (PTM Biolabs, SKU: PTM 315).

Statistical analysis: All statistical analyses were performed using GraphPad Prism 7 Software (California, USA). Data obtained from two or three individual experiments as mentioned in figure legends, were expressed as mean +/- SD. Either two-tailed unpaired Student's t-test or one-way ANOVA with Dunnett's/Bonferroni's multiple comparision was used to determine the statistical significance values. A Pvalue of equal to or less than 0.05 was considered statistically significant.

\section{Acknowledgments}

TKK is a recipient of JC Bose fellowship from Dept. of Science and Technology, Govt. of India (SR/S2/JCB-28/2010). AB is a senior research fellow of UGC. This work was supported by Life Science Education \& Training at JNCASR grant (DBT/INF/22/SP27679/2018). We acknowledge Dr. Rahul Gajbhiye from NIPER, Kolkata for the assistance in mass spectrometry related experiments in their central instrument facility. We thank Dr. R.G. Prakash for providing the necessary facilities to perform the mice experiment in Animal Facility, JNCASR, Dharaneeswar Reddy Manthri for technical assistance in animal experiment and the confocal facility, JNCASR for the immunofluorescence imaging. We also thank Kruthi H.T. for help in the design of the graphical abstract. We further acknowledge the Sophisticated Analytical Instrument Facility and Research (SAIFR, Lucknow) for providing spectral data. This manuscript bears CDRI communication number 2021/TK.

\section{References}

1. S. A.Haws, C. M. Leech and J. M. Denu, Metabolism and the Epigenome: A Dynamic Relationship. Trends Biochem 45, 731-747 (2020) 
2. Y. Chen et al. Lysine propionylation and butyrylation are novel post-translational modifications in histones. Mol Cell Proteomics 6, 812-819 (2007)

3. Z. Kaczmarska et al. Structure of p300 in complex with acyl-CoA variants. Nat Chem Biol 13, 2129 (2017)

4. E. J. Yoo, J. J. Chung, S. S. Choe, K. H. Kim, and J. B. Kim. Down-regulation of histone deacetylases stimulates adipocyte differentiation. J Biol Chem 281, 6608-6615 (2006)

5. J. L. Feldman, J. Baeza, and J. M. Denu. Activation of the protein deacetylase SIRT6 by longchain fatty acids and widespread deacylation by mammalian sirtuins. $J$ Biol Chem 288, 3135031356 (2013)

6. R. D. W. Kelly et al. Histone deacetylase (HDAC) 1 and 2 complexes regulate both histone acetylation and crotonylation in vivo. Sci Rep 8, 14690 (2018)

7. K. E. Wellen et al. ATP-citrate lyase links cellular metabolism to histone acetylation. Science 324, 1076-1080 (2009)

8. A. Goudarzi et al. Dynamic Competing Histone H4 K5K8 Acetylation and Butyrylation Are Hallmarks of Highly Active Gene Promoters. Mol Cell 62, 169-180 (2016)

9. M. T.King, and P. D.Reiss. Separation and measurement of short-chain coenzyme-A compounds in rat liver by reversed-phase high-performance liquid chromatography. Anal Biochem 146, 173179 (1985)

10. B. R. Sabari et al. Intracellular crotonyl-CoA stimulates transcription through p300-catalyzed histone crotonylation. Mol Cell 58, 203-215 (2015)

11. K. Mantelingu et al. Specific inhibition of p300-HAT alters global gene expression and represses HIV replication. Chem Biol 14, 645-657 (2007)

12. X. Liu et al. The structural basis of protein acetylation by the $\mathrm{p} 300 / \mathrm{CBP}$ transcriptional coactivator. Nature 451, 846-850 (2008)

13. Z. Xie et al.Metabolic Regulation of Gene Expression by Histone Lysine $\beta$-Hydroxybutyrylation. Mol Cell 62, 194-206 (2016)

14. R.Fellows et al. Microbiota derived short chain fatty acids promote histone crotonylation in the colon through histone deacetylases. Nat Commun 9, 105 (2018)

15. J. Wan, H. Liu, and L. Ming. Lysine crotonylation is involved in hepatocellular carcinoma progression. Biomed Pharmacother 111, 976-982 (2019)

16. G. Jiang et al. HIV latency is reversed by ACSS2-driven histone crotonylation. J Clin Invest 128, 1190-1198 (2018)

17. A. F. Kebede et al. Histone propionylation is a mark of active chromatin. Nat Struct Mol Biol 24, 1048-1056 (2017)

18. C. Gaviard, P. Cosette, T. Jouenne, and J.Hardouin. LasB and CbpD Virulence Factors of Pseudomonas aeruginosa Carry Multiple Post-Translational Modifications on Their Lysine Residues. J Proteome Res 18, 923-933 (2019)

19. K. Liu et al. A qualitative proteome-wide lysine crotonylation profiling of papaya (Carica papaya L.). Sci Rep 8, 8230 (2018)

20. S. Liu et al. Global Involvement of Lysine Crotonylation in Protein Modification and Transcription Regulation in Rice. Mol Cell Proteomics 17, 1922-1936 (2018)

21. Y. Lu et al. Dynamics and functional interplay of histone lysine butyrylation, crotonylation, and acetylation in rice under starvation and submergence. Genome Biol 19, 144 (2018)

22. O. K. Kwon, S. J. Kim and S.Lee. First profiling of lysine crotonylation of myofilament proteins and ribosomal proteins in zebrafish embryos. Sci Rep 8, 3652 (2018)

23. L. A. Farrelly et al. Histone serotonylation is a permissive modification that enhances TFIID binding to H3K4me3. Nature 567, 535-539(2019)

24. D. Zhang et al. Metabolic regulation of gene expression by histone lactylation. Nature $\mathbf{5 7 4 , 5 7 5 -}$ 580 (2019)

25. A. E. Lepack et al. Dopaminylation of histone $\mathrm{H} 3$ in ventral tegmental area regulates cocaine seeking. Science 368, 197-201 (2020)

26. A. Carrer et al. Impact of a High-fat Diet on Tissue Acyl-CoA and Histone Acetylation Levels. $J$ Biol Chem 292, 3312-3322 (2017) 
27. C. Bourgeuis et al. Specific Biological Features of Adipose Tissue, and their impact on HIV persistence. Front Microbiol 17 (2019)

28. C. Trapnell et al. Differential gene and transcript expression analysis of RNA-seq experiments with TopHat and Cufflinks. Nat Protoc 7, 562-578 (2012)

29. P. D. Thomaset al. PANTHER: a library of protein families and subfamilies indexed by function. Genome Res 13, 2129-2141 (2003)

30. M. J. L. de Hoon, S. Imoto, J. Nolan and S. Miyano. Open source clustering software. Bioinformatics 9, 1453-1454 (2004) 


\section{Figure 1}

a
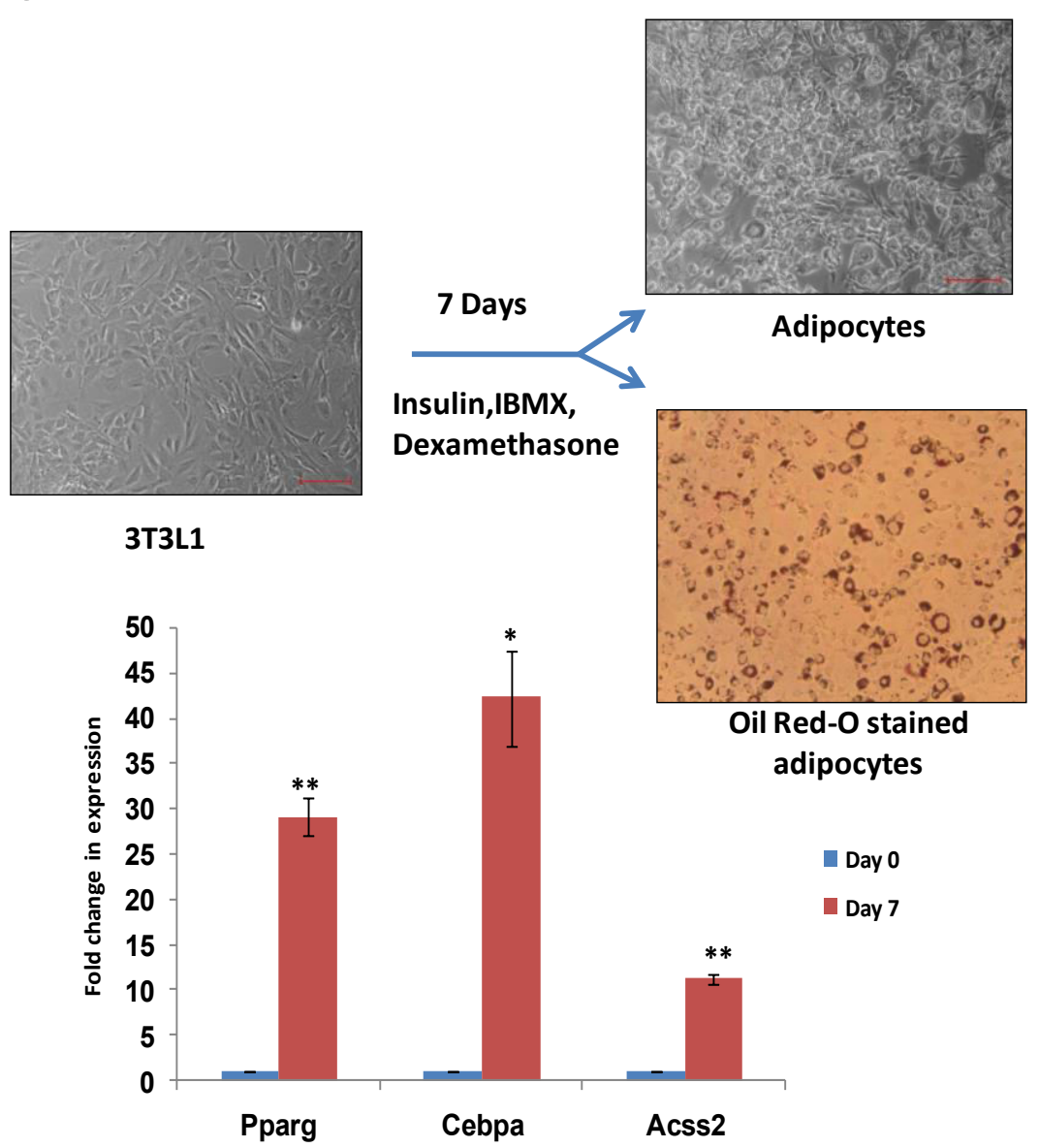

3T3L1

b

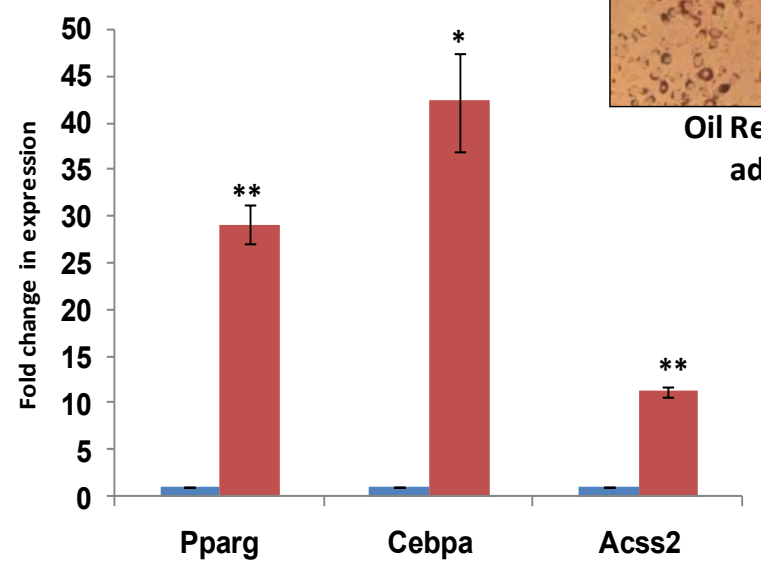

C

i.

$$
20 y^{0} D a y^{1} k D a
$$
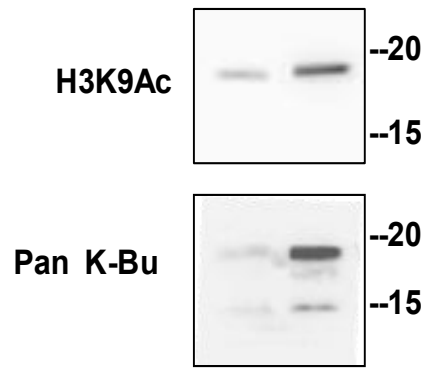

H3

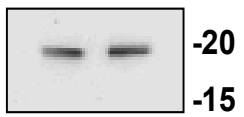

Direct blue

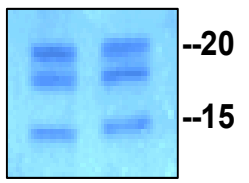

12 ii.
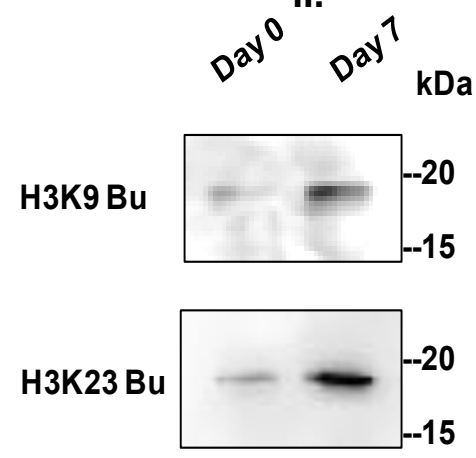

H4K8 Bu

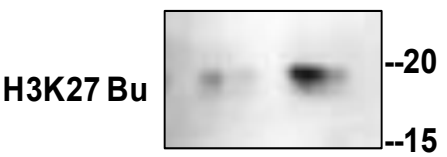

H4K12 Bu

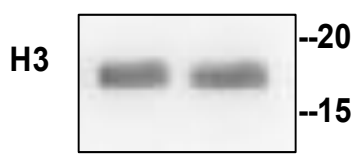

12
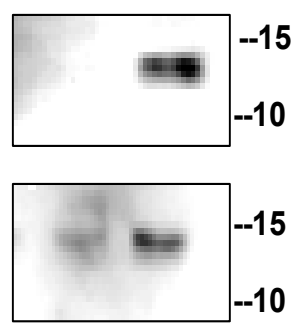

iii.
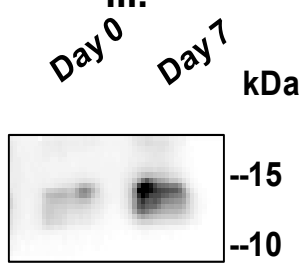
$-10$

H4

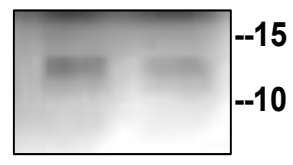

12 
Fig.1 Global histone butyrylation increases during adipogenesis: (a) Phase contrast images of 3T3L1 cells exhibiting fibroblast like morphology in their pre-adipocyte state and accumulation of lipid droplets in their adipocyte state. The lipid droplets can be stained using Oil Red-O dye. (b) Q-PCR analysis was performed to check for transcript levels of Acss2 in undifferentiated and differentiated 3T3L1 cells. Error bars denote mean $+/-\mathrm{SD}$ of three biological replicates; two-tailed unpaired Student's t-test: * $\mathrm{P}$ $<0.05,{ }^{* *} \mathrm{P}<0.01,{ }^{* * *} \mathrm{P}<0.001$, ns: not significant. (c) Histone acetylation and butyrylation levels in 3T3L 1 cells in undifferentiated and differentiated state were analysed by immunoblotting with antibodies specific for acetylated H3K9, butyrylated H3K9, H3K23, H3K27, H4K5, H4K8, H4K12 and pan-butyryl lysine antibodies. Antibodies against core histones $\mathrm{H} 3$ and $\mathrm{H} 4$ were used as loading controls along with Direct Blue staining of all four core histones. Lane 1- undifferentiated pre-adipocyte (Day 0) and Lane 2 differentiated adipocyte (Day 7 ) in each of the panels i, ii and iii. 


\section{Figure 2}

a i

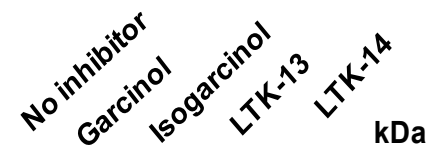

H3K18 Ac

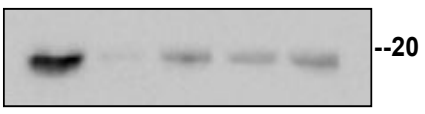

H3

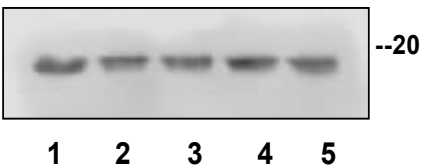

b i

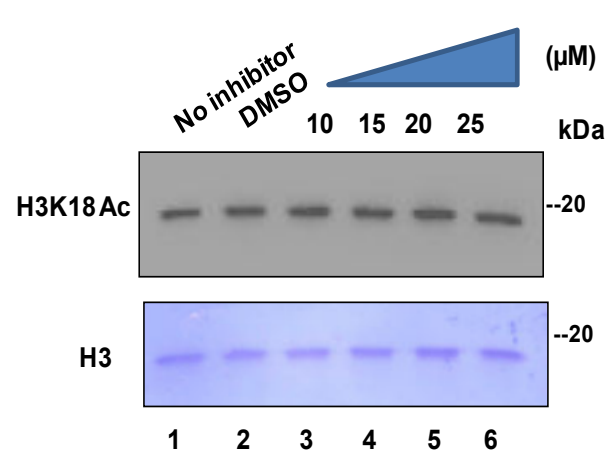

iii

H3K18 Ac

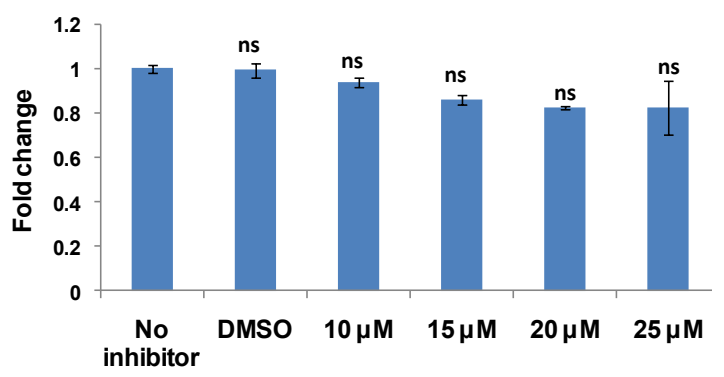

ii
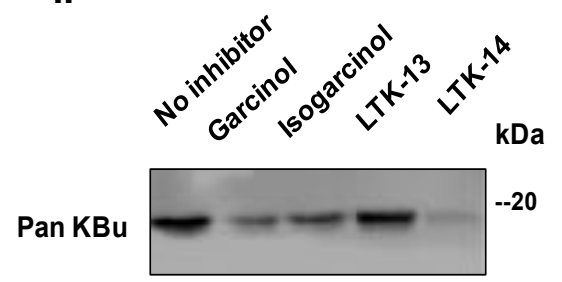

H3

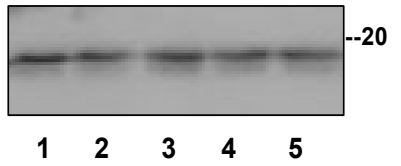

ii

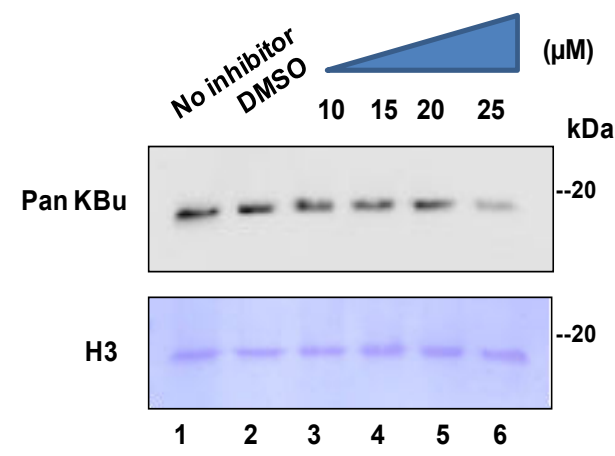

iv

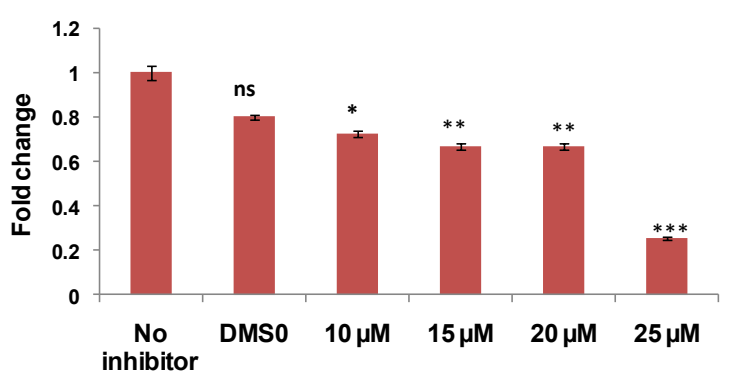


Fig. 2: Identification of a semi-synthetic small molecule that selectively inhibits p300 catalysed histone butyrylation in vitro: (a) Immunoblotting with antibodies against acetylated H3K18 and panbutyryl lysine to check for the relative inhibition of acetyltransferase and butyryltransferase activities of full length p300 by garcinol and its derivatives. Lane 1, enzyme alone; Lane 2, enzyme + garcinol; Lane 3, enzyme + isogarcinol; Lane 4, enzyme + LTK-13; Lane 5, enzyme + LTK-14. All the compounds were used at a concentration of $10 \mu \mathrm{M}$. Recombinant Xenopus histone $\mathrm{H} 3$ was used as loading control. (b) Immunoblotting with antibodies against acetylated H3K18 and pan-butyryl lysine to check for the relative inhibition of acetyltransferase and butyryltransferase activities of full length p300 by LTK-14A at different concentrations. Lane 1, enzyme alone; Lane 2, enzyme + DMSO; Lane 3-6, enzyme + LTK-14A at concentrations 10, 15, 20 and $25 \mu \mathrm{M}$ respectively. Images of Western blotting are given in panels $\mathrm{i}$ and ii and their quantitations are given in panels iii and iv. Error bars denote mean +/- SD of three biological replicates, one-way ANOVA with Bonferroni's multiple comparision; ${ }^{*} \mathrm{P}<0.05,{ }^{* *} \mathrm{P}<0.01,{ }^{* * *} \mathrm{P}<0.001$, ns: not significant. 


\section{Figure 3}

\section{a}

i.

LTK-14

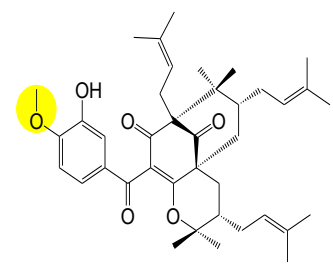

ii.

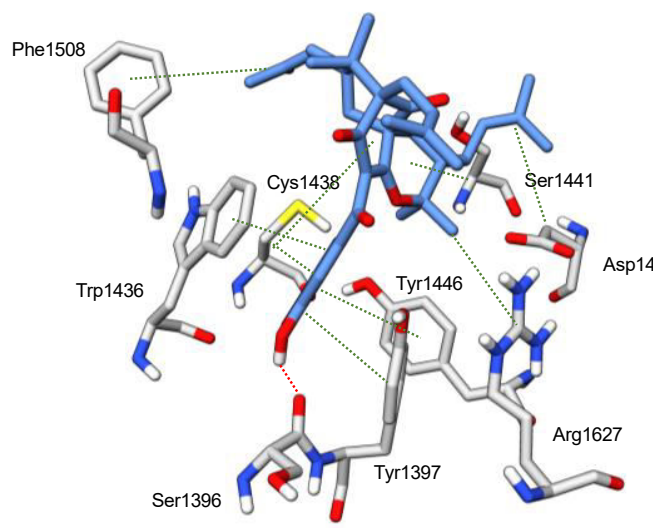

LTK-14 with p300 catalytic domain

b

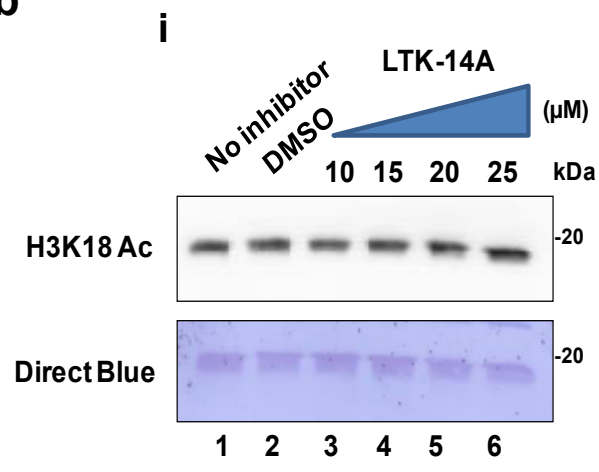

iii

H3K18 Ac

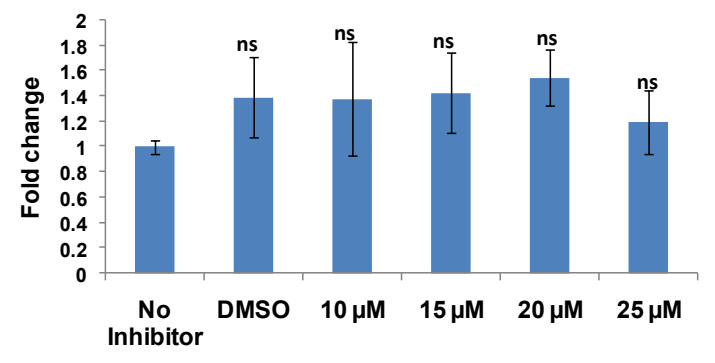

LTK-14A
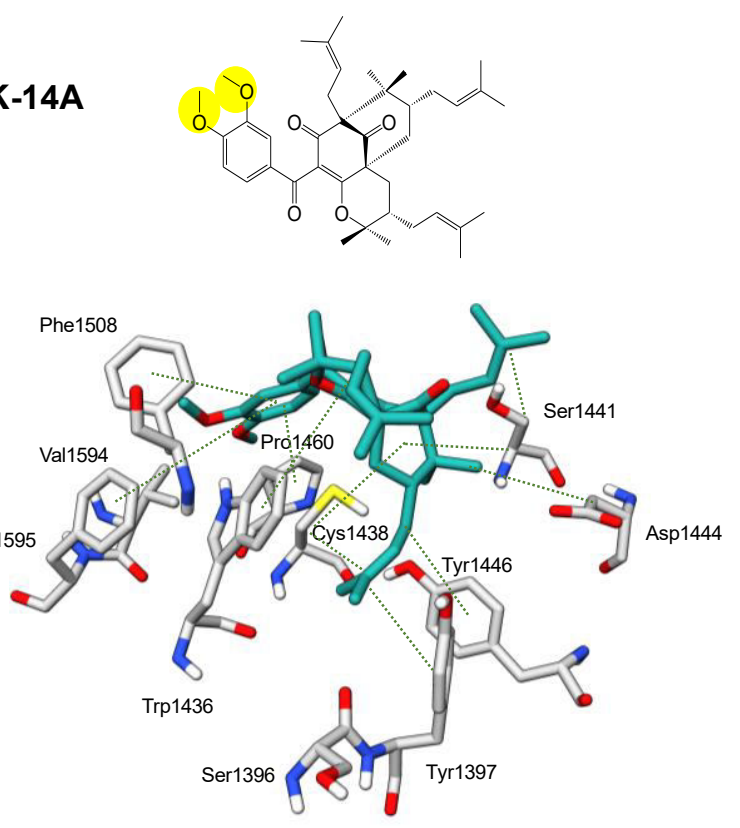

LTK-14A with p300 catalytic domain

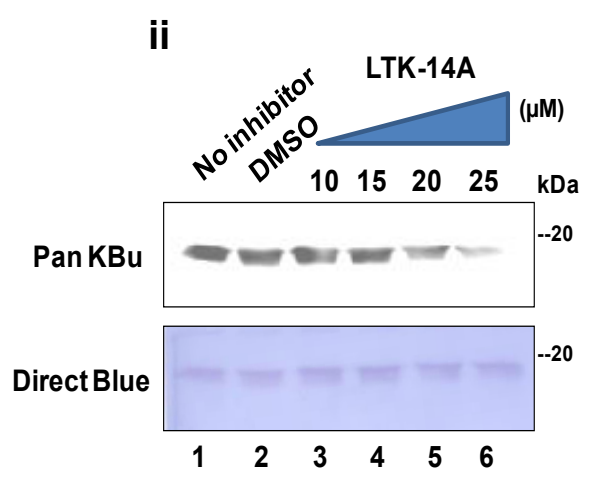

iv

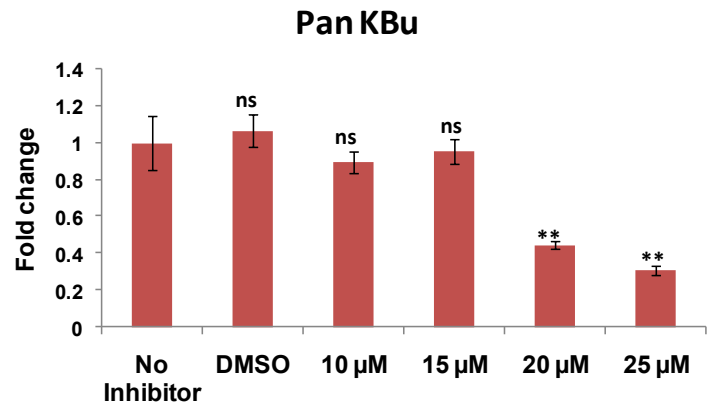




\section{Figure 3(..CONTINUED)}

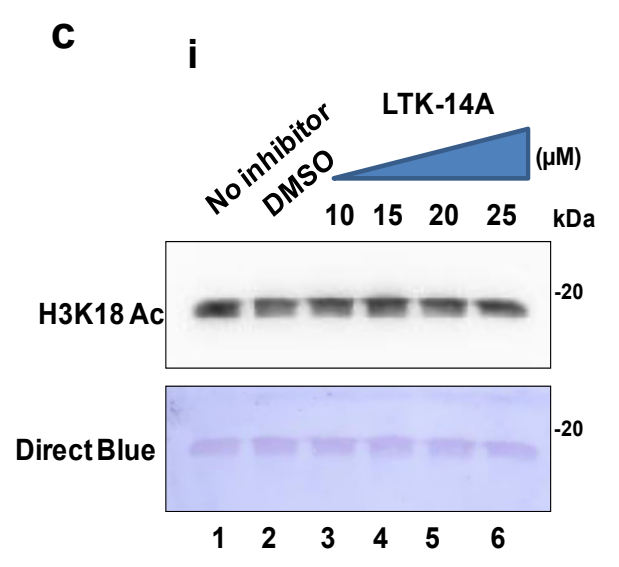

iii

H3K18 Ac

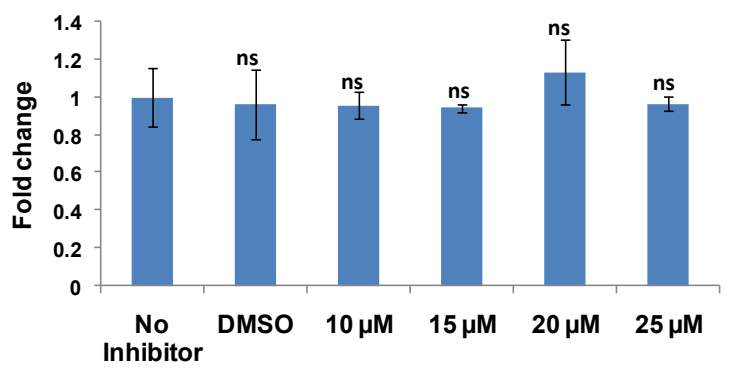

ii

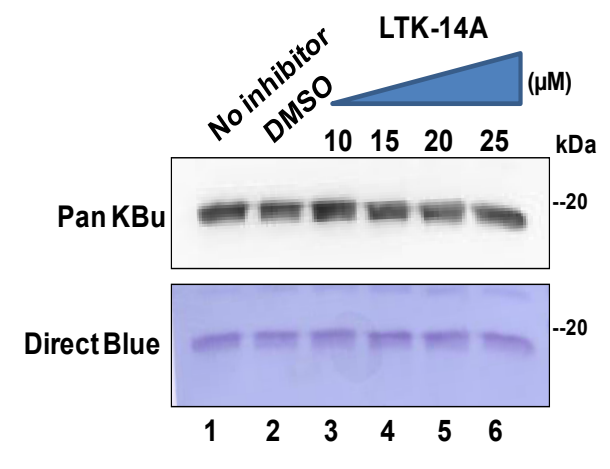

iv

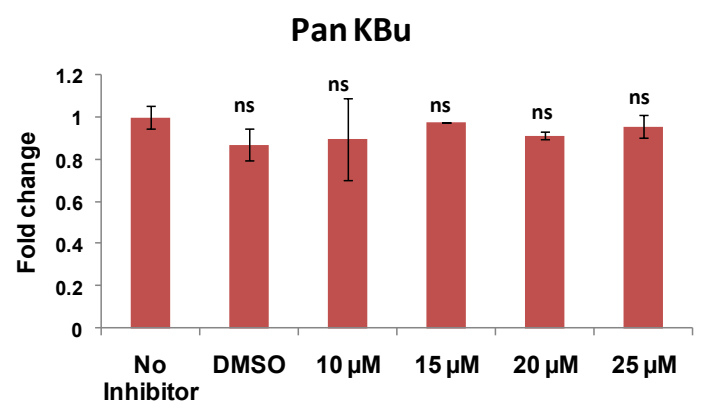


Figure 3:The critical interaction sites of the inhibitor in the catalytic domain for p300 mediated butyrylation: (a) Molecular structures of mono- (LTK-14) and di-(LTK-14A) substituted isogarcinol (panel i). Molecular docking studies for studying comparative interaction of LTK-14 and LTK-14A with the amino acid residues of p300 catalytic domain. The predicted interactions are denoted by tick lines (panel ii). Hydrogen bonding is shown in red and hydrophobic interaction is shown in green. (b) Immunoblotting with antibodies against acetylated H3K18 and pan-butyryl lysine to check for the relative inhibition of acetyltransferase and butyryltransferase activities of wild type p300 catalytic domain by LTK-14A at different concentrations. Lane 1, enzyme alone; Lane 2, enzyme + DMSO; Lane 3-6, enzyme + LTK-14A at concentrations 10,15, 20 and $25 \mu \mathrm{M}$ respectively. Images of Western blotting are given in panels $\mathrm{i}$ and ii and their quantitations are given in panels iii and iv. Error bars denote mean +/- SD of three biological replicates, one-way ANOVA with Bonferroni's multiple comparison; ${ }^{*} \mathrm{P}<0.05$, ${ }^{* *} \mathrm{P}<0.01$, ${ }^{* * *} \mathrm{P}<0.001$, ns: not significant. (c) Immunoblotting with antibodies against acetylated H3K18 and pan-butyryl lysine to check for the relative inhibition of acetyltransferase and butyryltransferase activities of catalytically active p300 catalytic domain with point mutation C1438A by LTK-14A at different concentrations. Lane 1, enzyme alone; Lane 2, enzyme + DMSO; Lane 3-6, enzyme + LTK-14A at concentrations 10, 15, 20 and $25 \mu \mathrm{M}$ respectively. Images of Western blotting are given in panels $\mathrm{i}$ and ii and their quantitation are given in panels iii and iv. Error bars denote mean +/- SD of three biological replicates, one-way ANOVA with Bonferroni's multiple comparison; ${ }^{*} \mathrm{P}<0.05,{ }^{* *} \mathrm{P}<0.01$, ${ }^{* *} \mathrm{P}<0.001$, ns: not significant. 


\section{Figure 4}

a

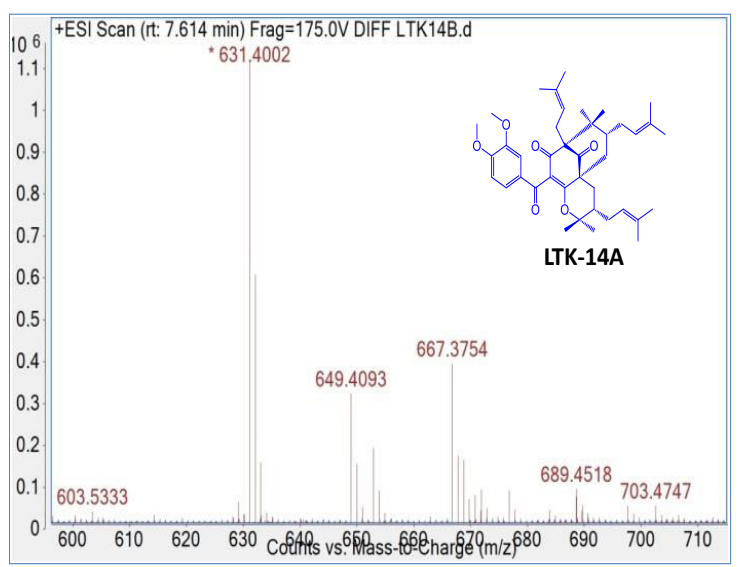

C

i.

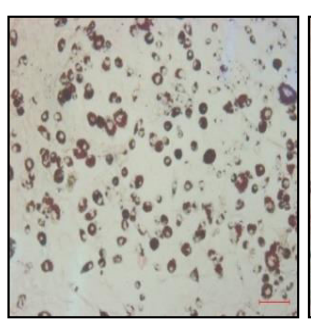

No inhibitor

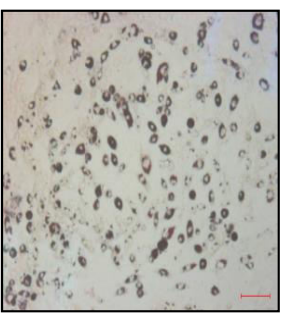

DMSO

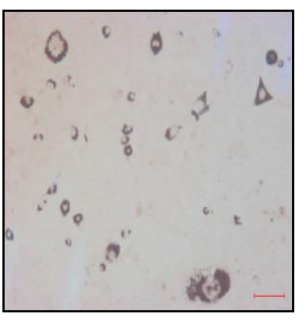

LTK14A
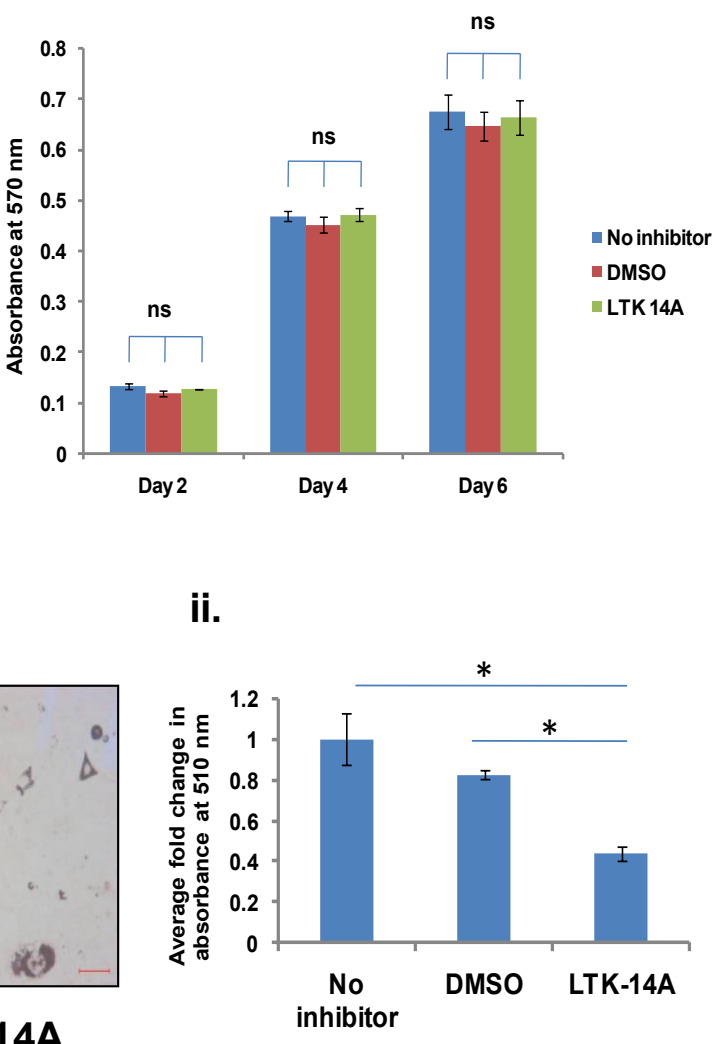

ii. ii.

b

inhibitor i.

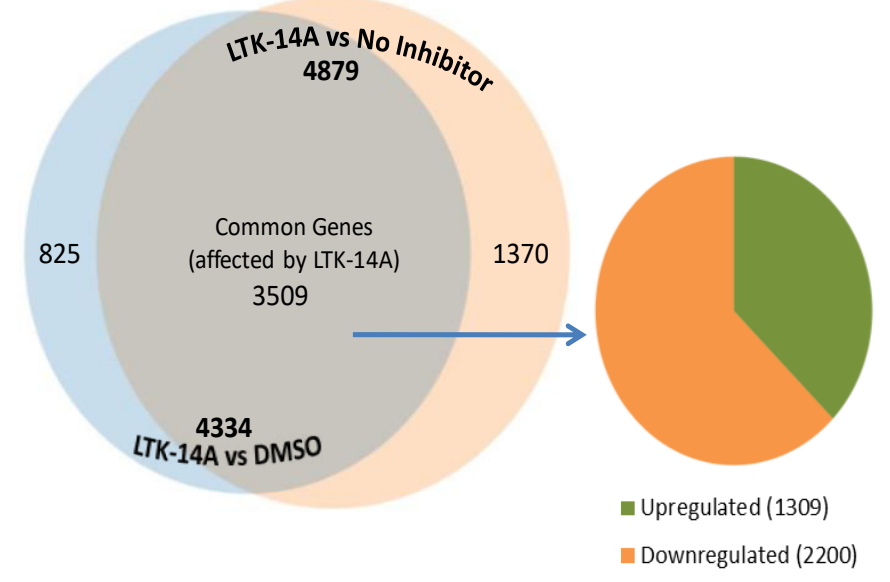

d

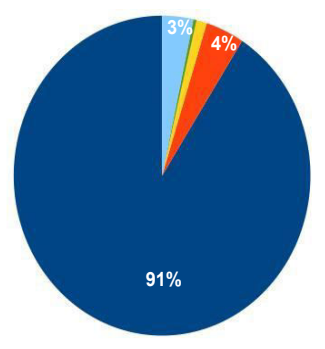

In Protein coding

= InCRNA

Erocessed pseudogene

- Transcribed unprocessed pseudogene

- transcribed_unitary_pseudogene

Enclassified 
Fig. 4: The butyrylation inhibitor represses adipogenesis in 3T3L1 cells: (a) The permeability and stability of LTK-14A in 3T3L1 was tested by LC-ESI-MS analysis of metabolites, extracted from the compound treated 3T3L1 cells to detect the protonated LTK-14A in the ESI spectra of the eluted fraction. (b) MTT assay was performed to check for any possible cellular toxicity effect of LTK-14A, using $25 \mu \mathrm{M}$ concentration of the compound on 3T3L1 cells upon incubation for varying lengths of time $(2,4$ and 6 days). Comparison was done with cells that were left untreated or treated with DMSO as solvent control. (c) The effect of LTK-14A (25 $\mu \mathrm{M})$ treatment on adipogenesis was tested by Oil Red-O staining of the adipocytes (panel $\mathrm{i}$ ) and the quantitation of staining is represented graphically in panel ii. Error bars denote mean +/- SD of three biological replicates,one-way ANOVA with Bonferroni's multiple comparison; ${ }^{*} \mathrm{P}<0.05$, ${ }^{* *} \mathrm{P}<0.01$, ${ }^{* * *} \mathrm{P}<0.001$, ns: not significant. (d) The effect of LTK-14A on the global transcription network inside 3T3L1 cells was investigated by high throughput transcriptome analysis with RNA isolated from compound treated, DMSO treated and untreated 3T3L 1 cells $(n=2$ in each case). Venn diagram showing the number of differentially expressed genes upon LTK-14A treatment with respect to untreated and DMSO treated conditions (panel i). Classification of the common differentially expressed genes according to their type (panel ii). 


\section{Figure 5}

a

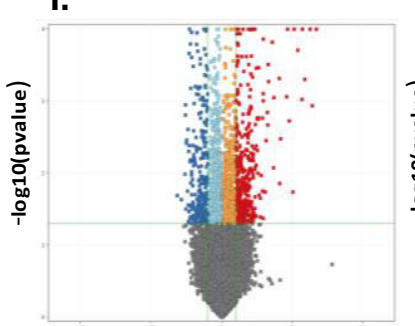

b

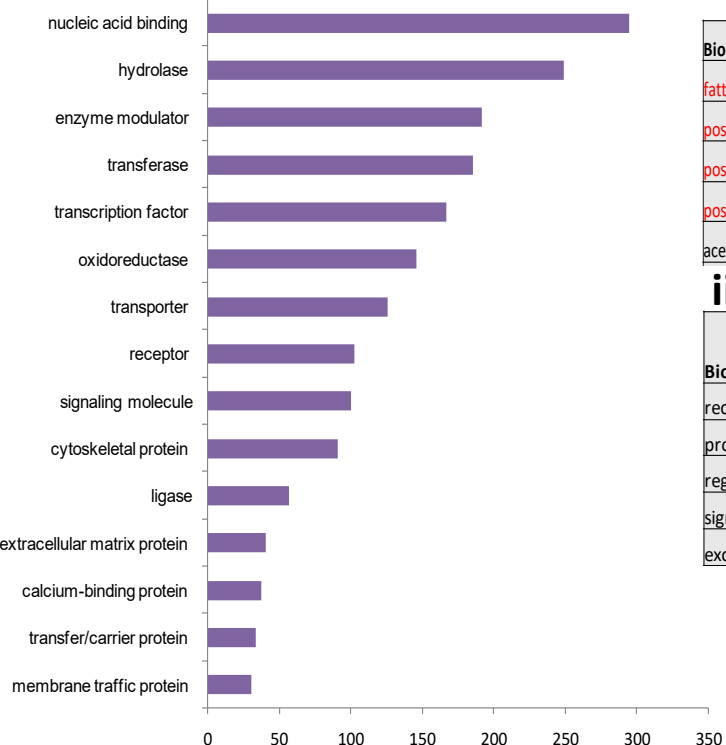

c. i.

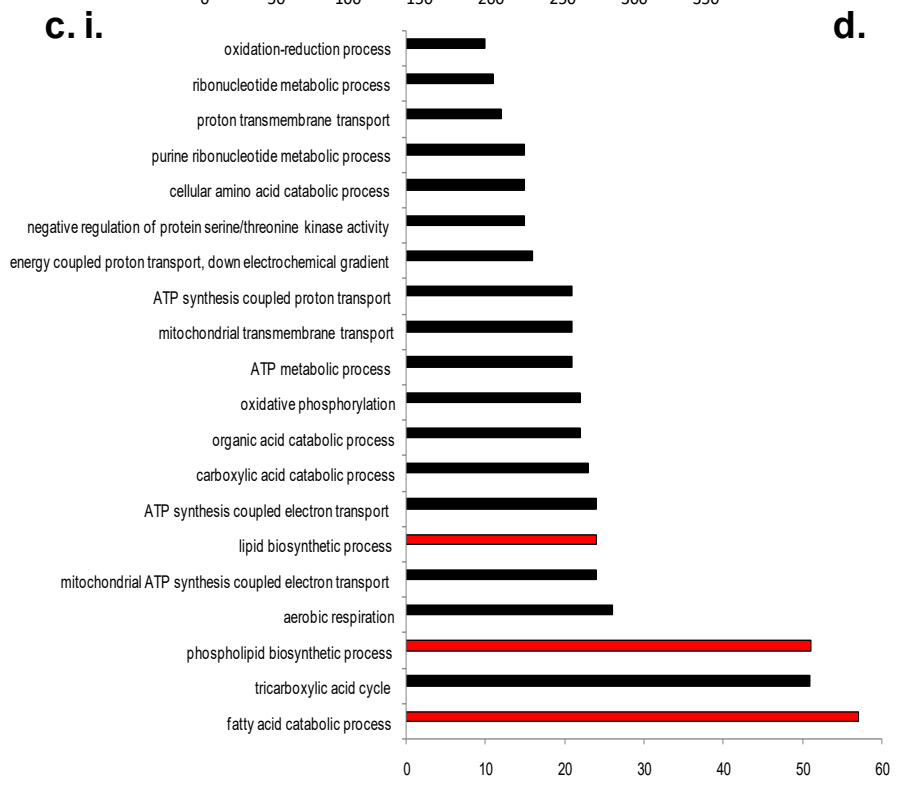

ii.

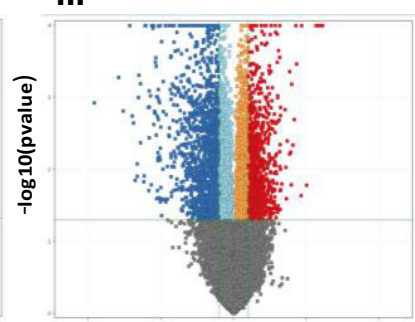

$\log 2$ (fold change)

c. ii. iii.

.

iii.

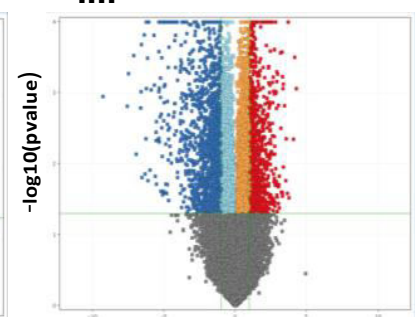

$\log 2$ (fold change)

\begin{tabular}{|l|r|r|r|}
\hline Biological processes & Fold enrichment & P-value & \multicolumn{1}{c|}{ FDR } \\
\hline fatty acid beta-oxidation using acy-COA dehydrogenase & 6.93 & 0.0000556 & 0.00304 \\
\hline positive regulation of cholesterol biosynthetic process & 6.06 & 0.00129 & 0.0442 \\
\hline positive regulation of cholesterol metabolic process & 6.06 & 0.00129 & 0.0441 \\
\hline positive regulation of sterol biosynthetic process & 6.06 & 0.00129 & 0.044 \\
\hline acetyl-CoA biosynthetic process from pyruvate & 6.06 & 0.00129 & 0.0439 \\
\hline
\end{tabular}

\begin{tabular}{|l|r|r|r|}
\hline & & & \multicolumn{1}{|l|}{} \\
\hline Biological process & Fold enrichment & P-value & \multicolumn{1}{l|}{ FDR } \\
\hline receptor localization to synapse & 4.78 & 0.0000625 & 0.0448 \\
\hline protein localization to synapse & 4.37 & 0.0000078 & 0.00724 \\
\hline regulation of synaptic plasticity & 3.55 & $1.25 E-09$ & 0.00000393 \\
\hline signal release & 2.97 & 0.0000189 & 0.0166 \\
\hline exocytosis & 2.83 & 0.00000493 & 0.00519 \\
\hline
\end{tabular}
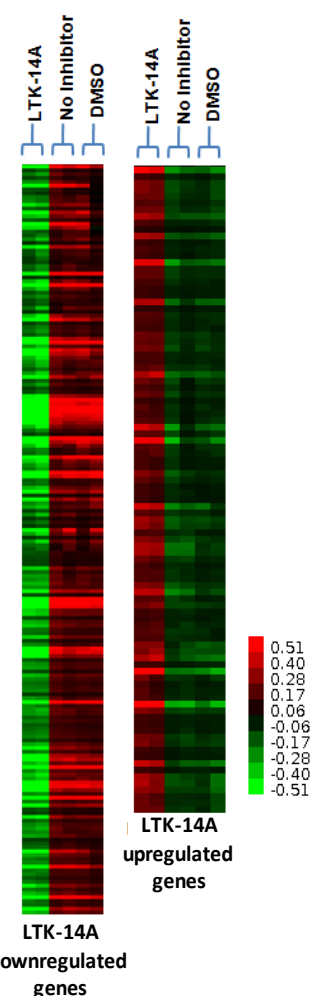
Figure 5(...continued)

e.
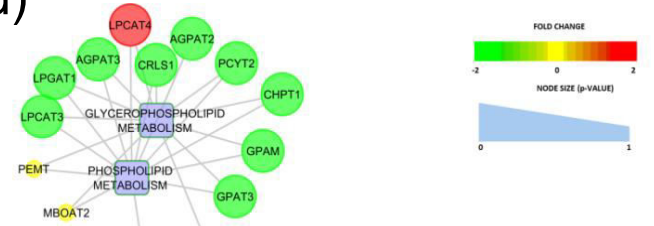

MBOAT2

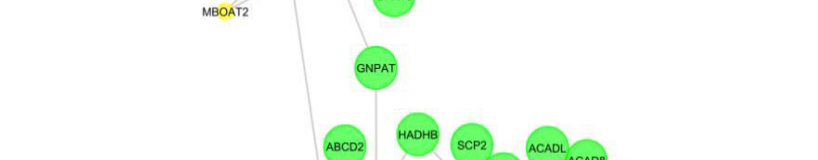

\section{f.}

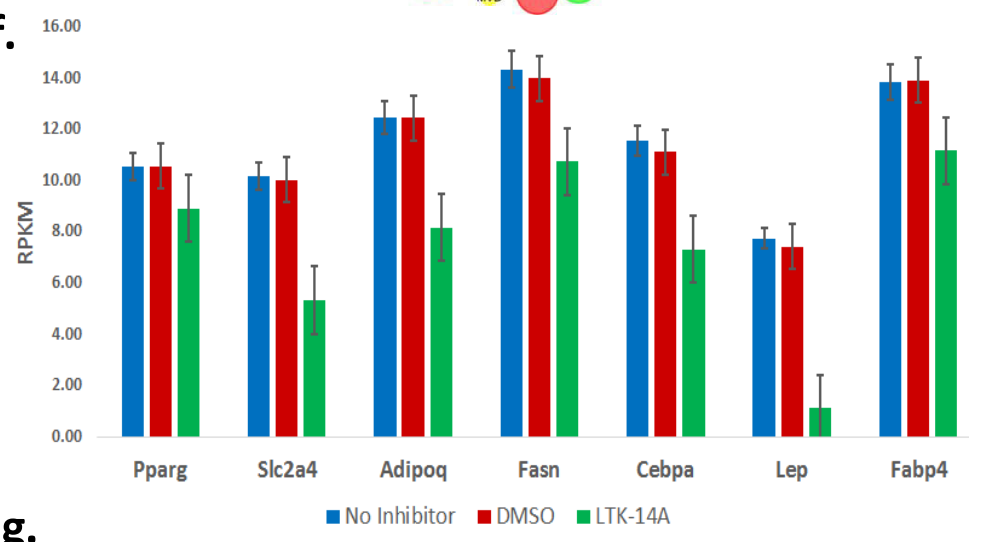

g.

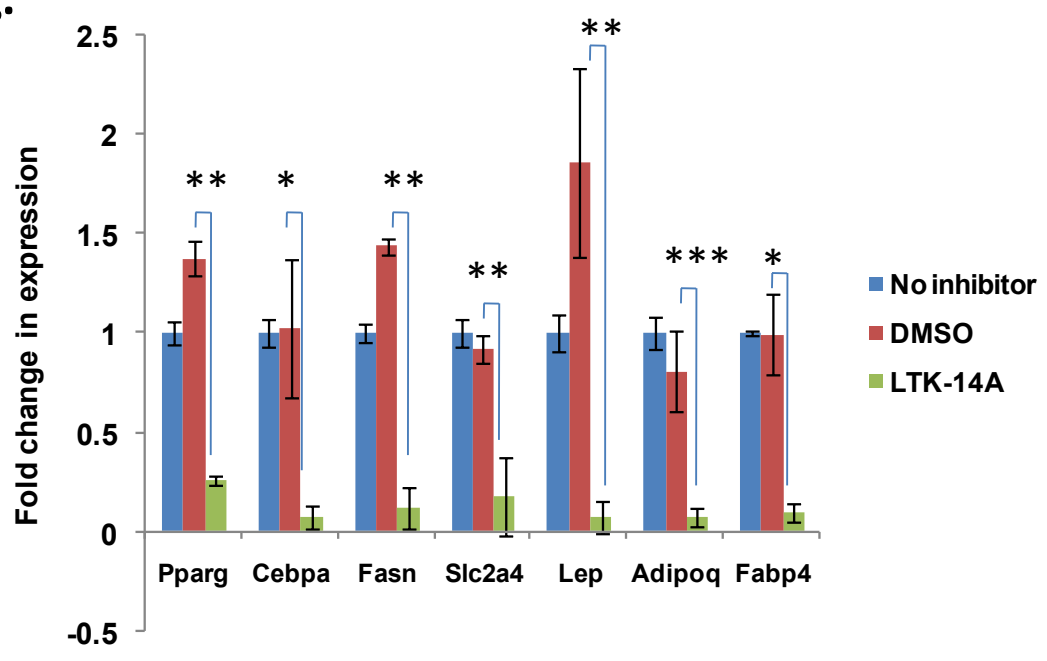


bioRxiv preprint doi: https://doi.org/10.1101/2021.08.01.454641; this version posted August 1, 2021. The copyright holder for this preprint (which was not certified by peer review) is the author/funder. All rights reserved. No reuse allowed without permission.

Figure 5: The p300 butyrylation inhibitor, LTK-14A represses expression of several pro-adipogenic genes and inhibits lipid metabolism: (a) Differential gene expression pattern of three conditions compared with each other, depicted in volcano plots- No Inhibitor versus DMSO (panel i), No Inhibitor versus LTK-14A (panel ii) and DMSO versus LTK-14A (panel iii). (b) Classification of the differentially expressed protein coding genes upon LTK-14A treatment according to their functionality. (c) Gene ontology analysis was performed to study the different biological pathways that were affected by LTK-14A in 3T3L1 compared to DMSO treated as well as untreated conditions, as predicted by Panther software. The pathways were ranked in the order of those with maximum number of genes affected (panel i). A different ranking order was created on the basis of fold enrichment i.e. the pathways in which the ratio of number of observed affected genes over number of expected genes to be affected was the highest (panel ii). A similar analysis was also performed for genes differentially expressed in DMSO versus untreated conditions (panel iii). (d) Heat maps showing the differential gene expression pattern between LTK-14A treated cells and DMSO treated or untreated cells. (e) A molecular network was constructed to depict the interconnectivity between the lipid metabolism related genes and pathways that were affected upon their dysregulation by LTK-14A compared to DMSO treated conditions.(f) Pro-adipogenic genes that could be putative targets for LTK-14A were identified and RPKM values for these genes have been depicted graphically. (g) Q-PCR analysis was performed to validate the putative target genes of LTK-14A. Error bars denote mean +/- SD of three biological replicates; one-way ANOVA with Bonferroni's multiple comparison: ${ }^{*} \mathrm{P}<0.05,{ }^{* *} \mathrm{P}<0.01,{ }^{* *} \mathrm{P}<0.001$, ns: not significant. 


\section{Figure 6}
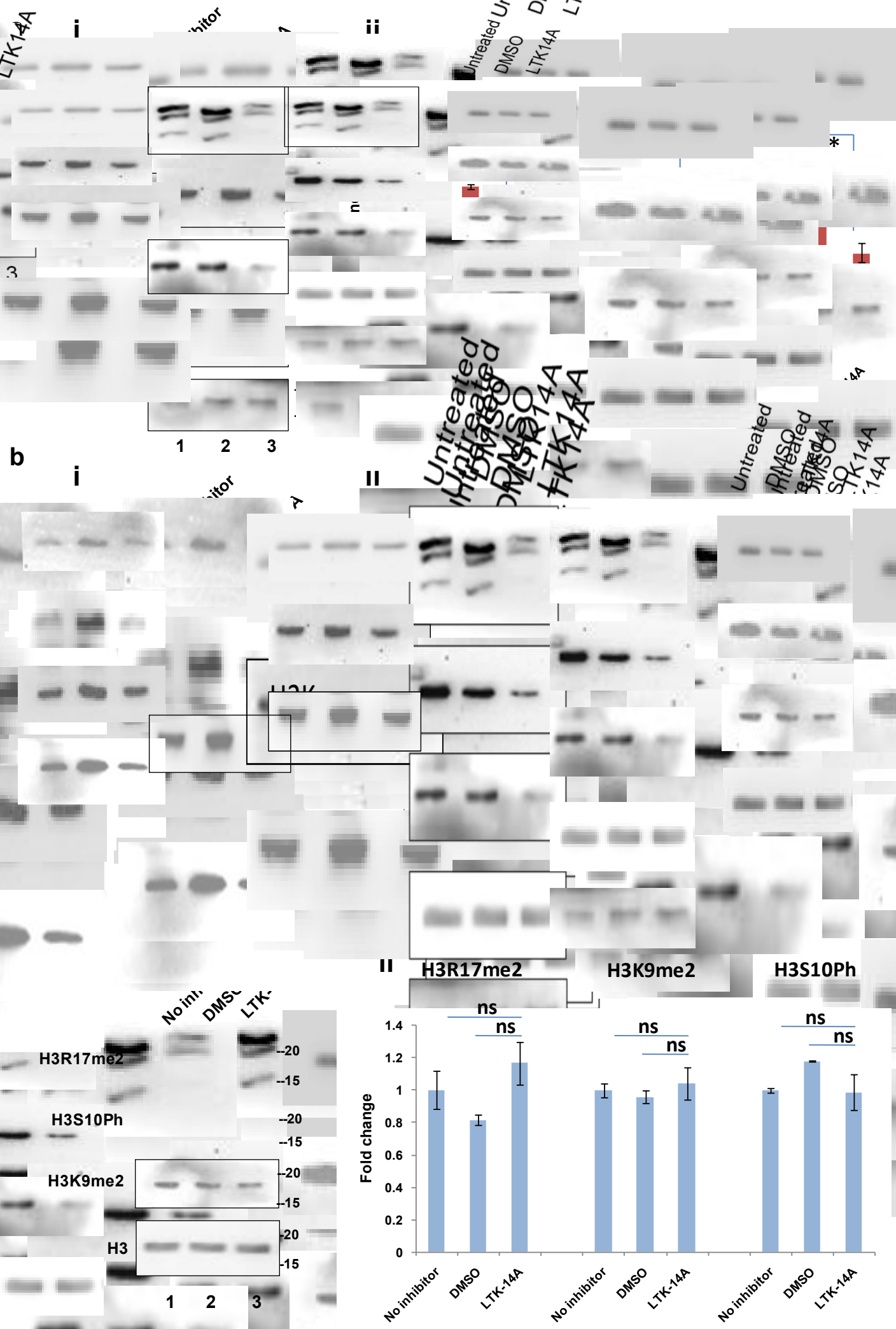
Fig.6: LTK-14A inhibits histone butyrylation in 3T3L1 cells, without affecting acetylation or other post-translational modifications: (a) Histone butyrylation levels in 3T3L1 cells that were treated with LTK-14A were compared with that in DMSO treated and untreated conditions by immunoblotting with antibodies against butyrylated histone $\mathrm{H} 3 \mathrm{~K} 23, \mathrm{H} 4 \mathrm{~K} 5$ and pan-butyryl lysine. Histones $\mathrm{H} 3$ and $\mathrm{H} 4$ were used as loading controls (panel i). The fold change of quantified band intensity is graphically represented in panel (ii) in which the error bars denote mean +/- SD of three biological replicates.(b) Under similar conditions histone acetylation levels were compared by immunoblotting using antibodies against acetylated histones $\mathrm{H} 3 \mathrm{~K} 18, \mathrm{H} 3 \mathrm{~K} 9$ and $\mathrm{H} 4 \mathrm{~K} 12$. The result is graphically represented in panel (ii) in which error bars denote mean $+/$ - SD of three biological replicates. (c) Immunoblotting was also performed with antibodies against histone H3R17 dimethylation, H3S10 Phosphorylation and H3K9 dimethylation. The result is graphically represented in panel (ii) in which error bars denote mean +/- SD of three biological replicates. For all data one-way ANOVA with Bonferroni's multiple comparision was performed: * $\mathrm{P}<$ $0.05,{ }^{* *} \mathrm{P}<0.01,{ }^{* * *} \mathrm{P}<0.001$, ns: not significant . 


\section{Figure 7}

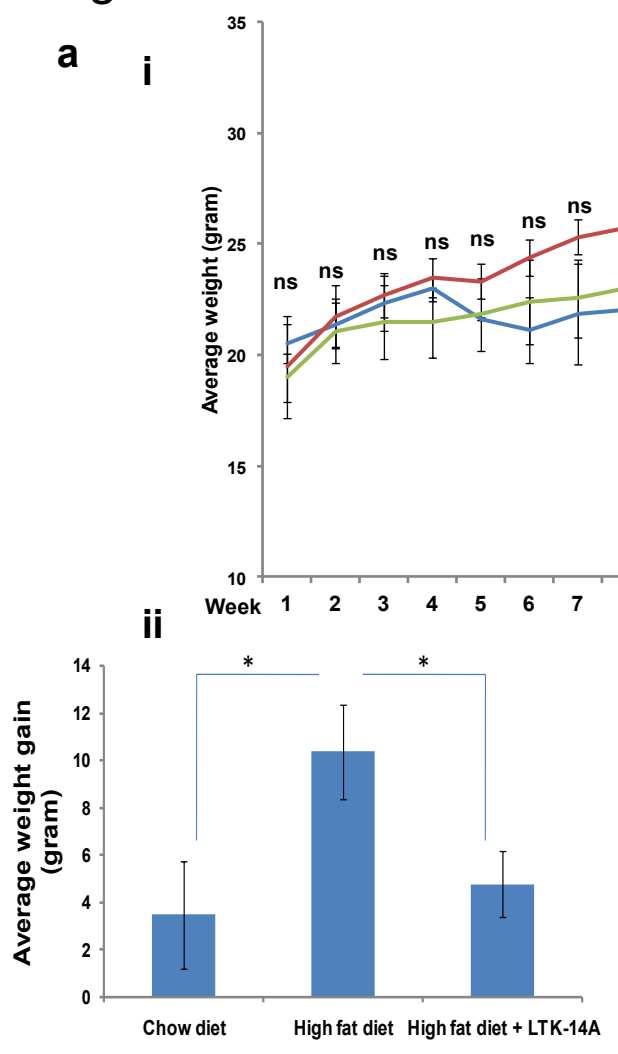

ii

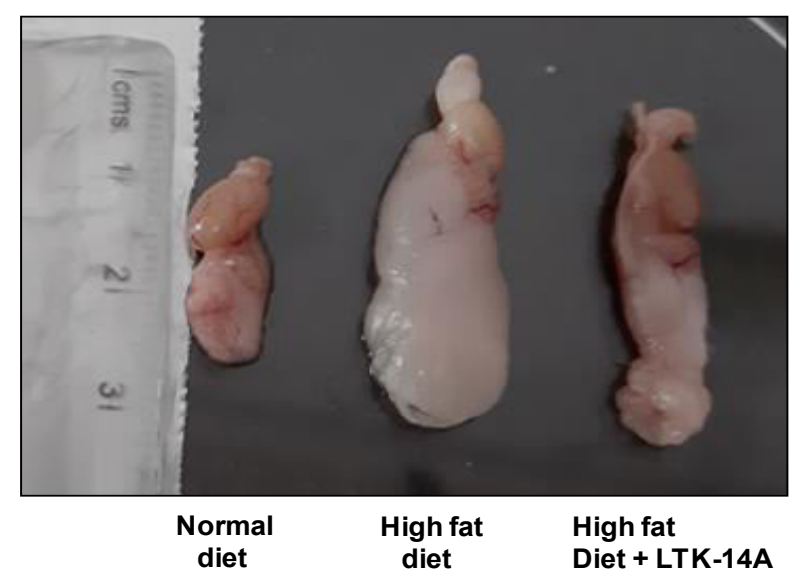

c.i

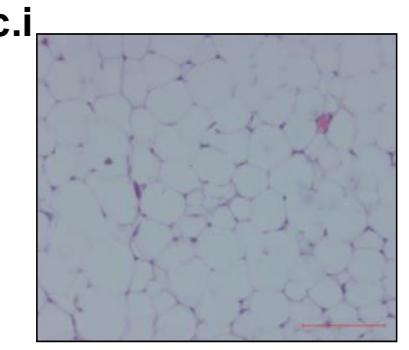

Normal diet

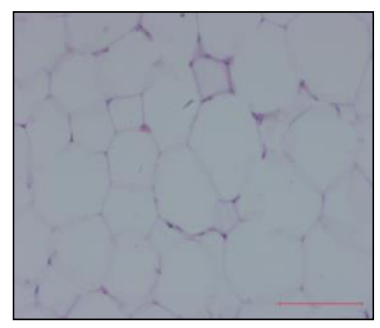

High fat diet
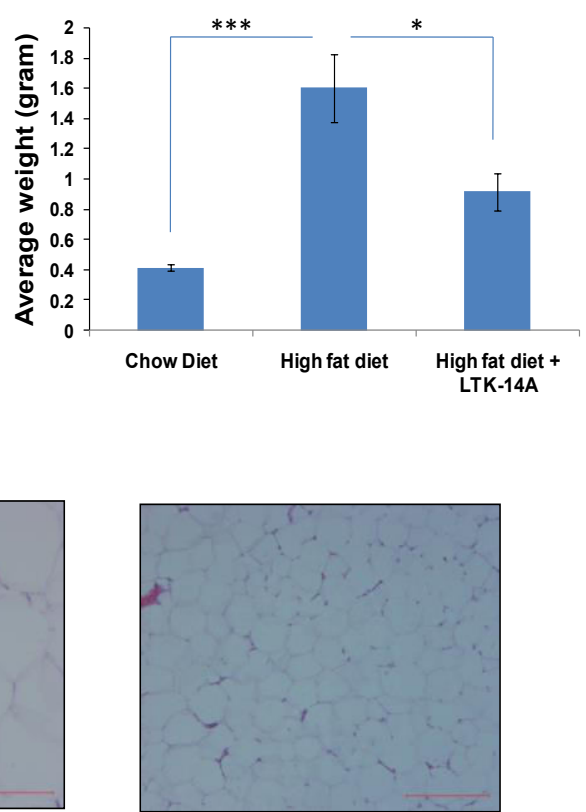

High fat diet + LTK-14A 
Figure $7(\ldots$ continued $)$

ii

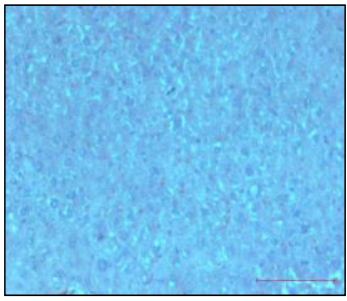

Normal diet

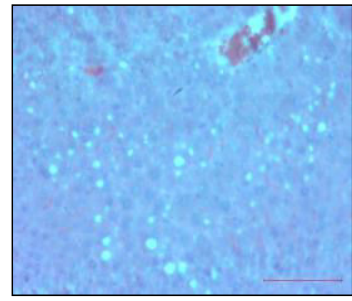

High fat diet

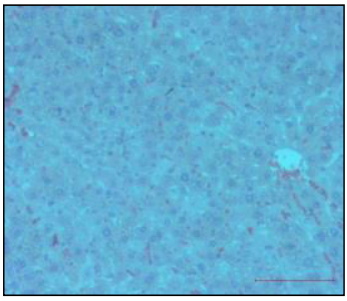

High fat diet + LTK -14A

d i

ii

iii
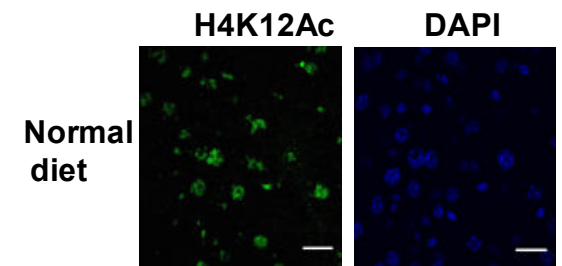

H3K14 Ac

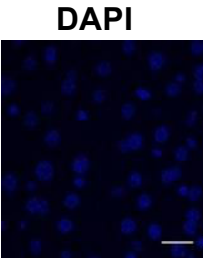

$\mathrm{H} 4 \mathrm{~K} 5 \mathrm{Bu}$

DAPI
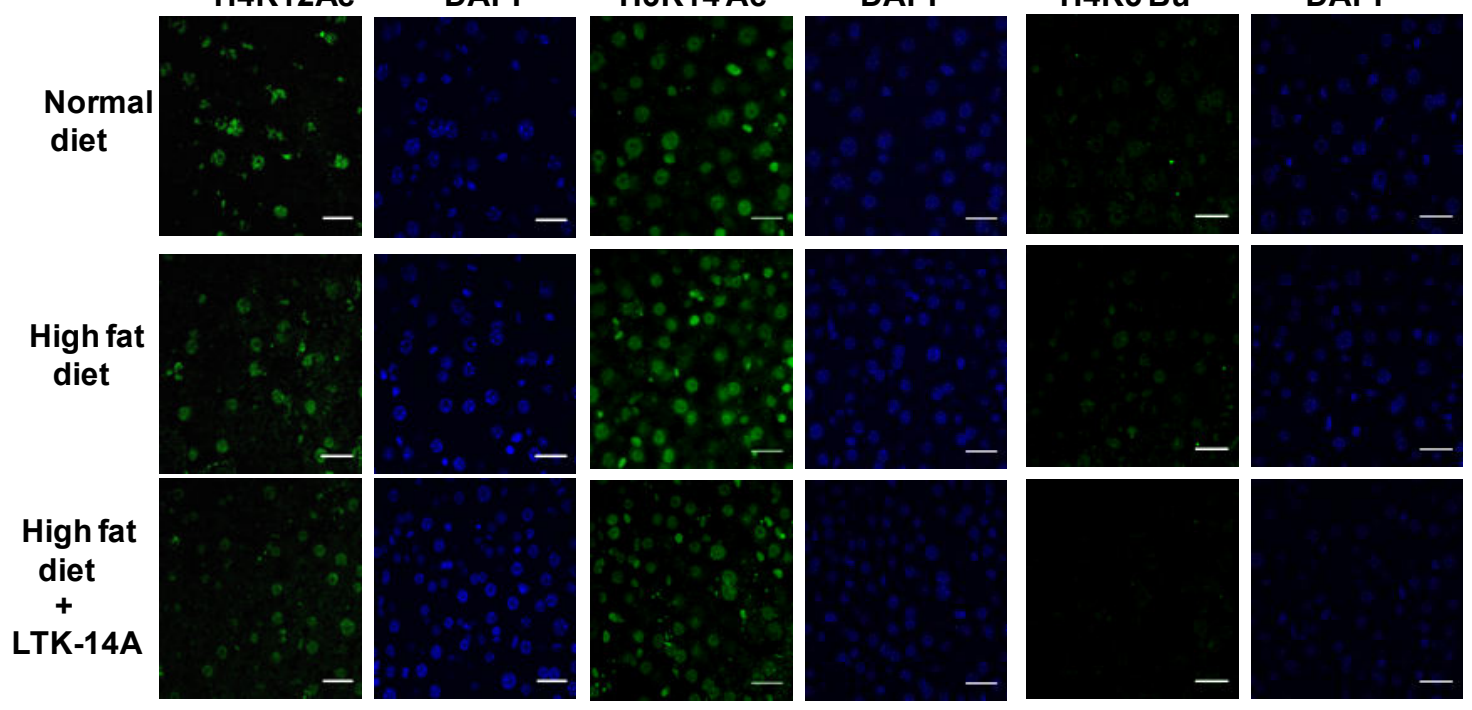

iv
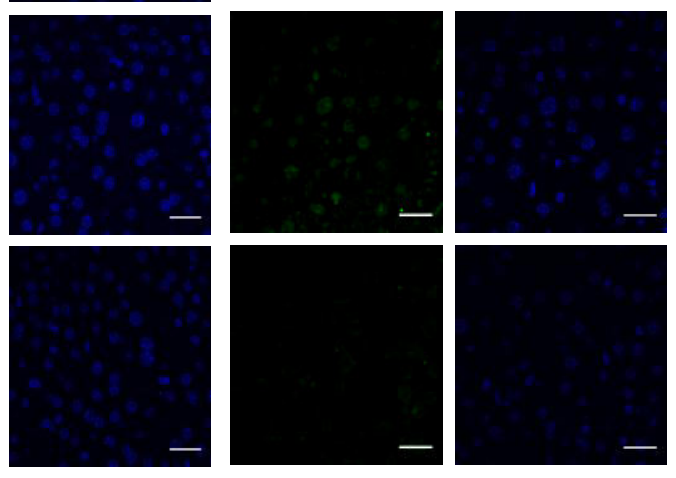

v
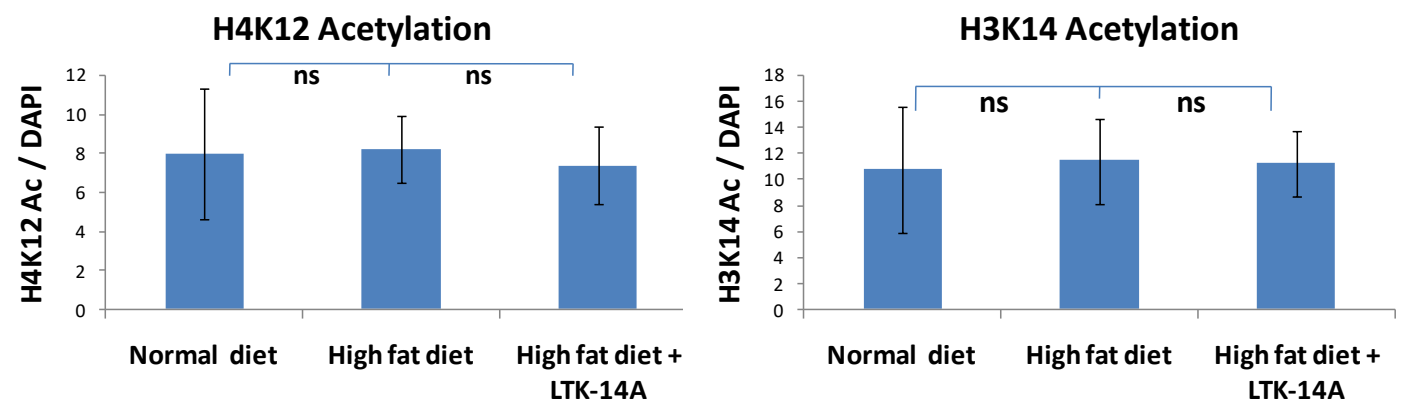
Figure 7 (...continued)

Vi

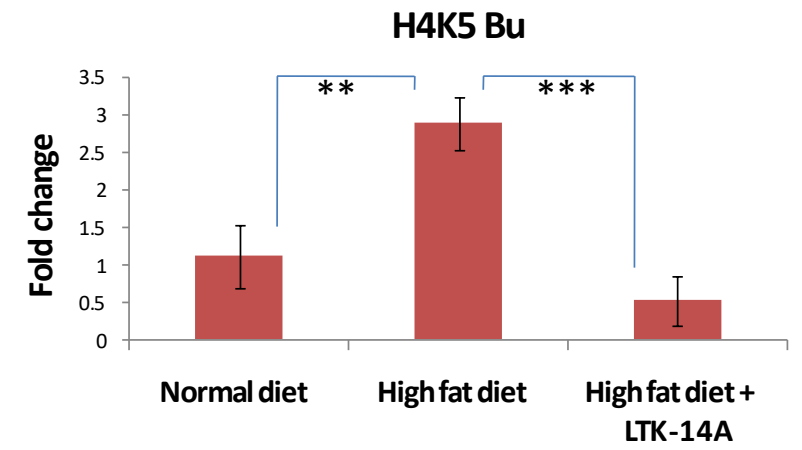

e

\section{Replicate $1 \quad$ Replicate 2}

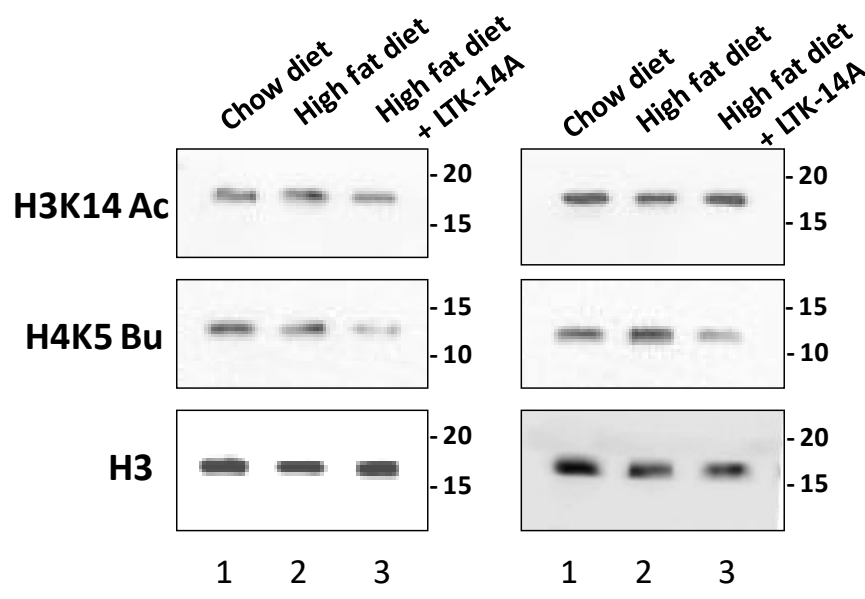

ii

\section{H3K14 AC}

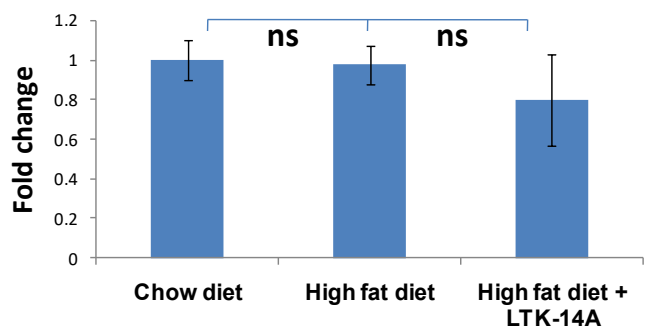

iii

H4K5 Bu

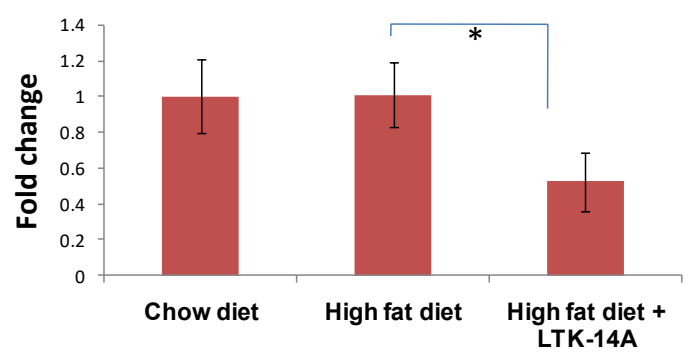


bioRxiv preprint doi: https://doi.org/10.1101/2021.08.01.454641; this version posted August 1, 2021. The copyright holder for this preprint (which was not certified by peer review) is the author/funder. All rights reserved. No reuse allowed without permission.

\section{Figure 7 (..continued)}

\section{f}

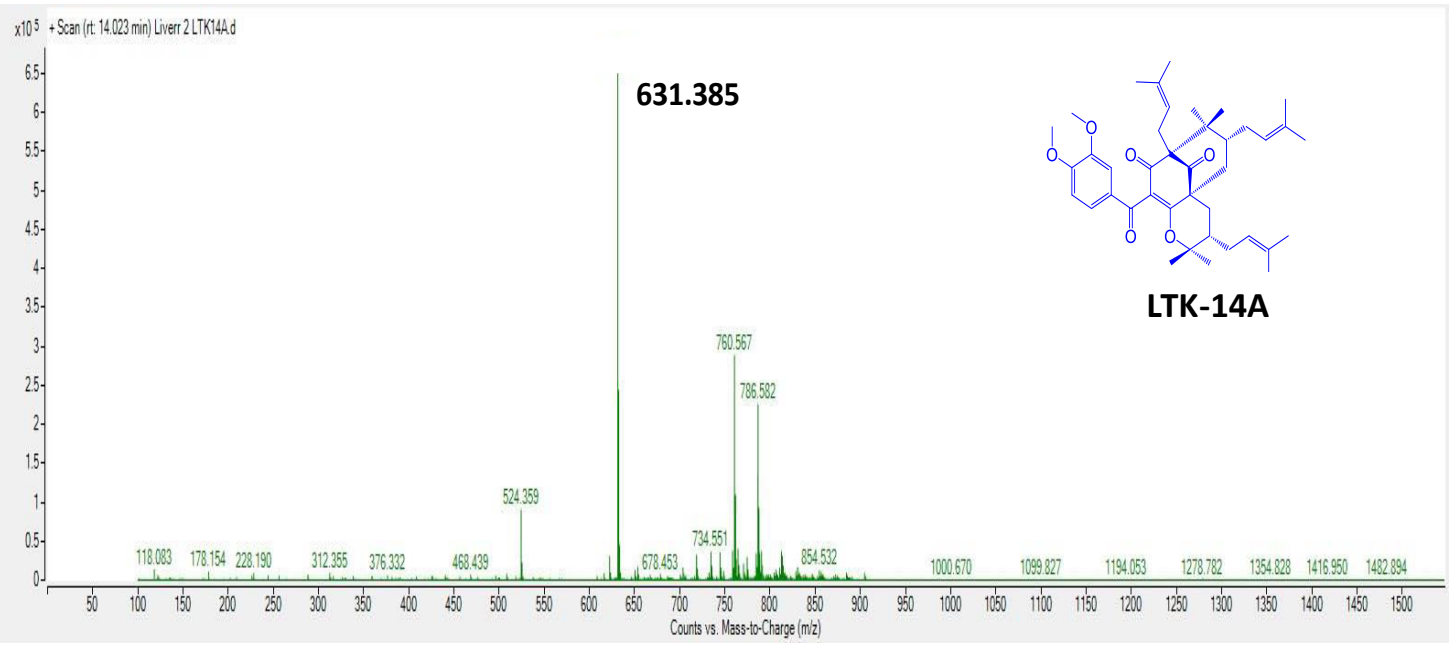


Fig. 7: The weight ganing of high fat diet fed mice (C57BL6/J) could be significantly reduced $b$ the butyrylation specific inhibitor of p300: (a) Trend of weight gaining (panel i) and average body weight gained after 16 weeks (panel ii) of C57BL6/J mice maintained on normal chow diet, high fat diet and high fat diet mixed with LTK-14A was plotted ; $n=4$ in each group. (b) Representative images of dissected bodies showing fat accumulation (panel $i$ ) and epididymal fat pads (panel ii) of the mice from three groups. The average weight of the fat pads is represented graphically (panel iii); $n=4$ in each group. (c) Representative images of morphology of adipose tissue (panel i) and liver (panel ii) from the mice of the three groups, as studied by hematoxylin and eosin staining. For all data one-way ANOVA with Dunnett's multiple comparision was performed: ${ }^{*} P<0.05$, ${ }^{* *} P<0.01$, ${ }^{* * *} P<0.001$, ns: not significant.(d) Immunofluorescence microscopy of liver sections stained with antibodies against histone H4K12 acetylation (panel i), histone H3K14 acetylation (panel ii) and histone H4K5 butyrylation (panel iii). The fluorescence intensity of the modified histone marks was normalised against DAPI staining of whole nuclei for quantitation which is graphically depicted in panel iv, $v$ and $v i ; n=4$ in each group; one-way ANOVA with Dunnett's multiple comparision: ${ }^{*} \mathrm{P}<0.05$, ${ }^{* *} \mathrm{P}<0.01$, ${ }^{* * *} \mathrm{P}<0.001$, ns: not significant. (e) Histone H3K14 acetylation and H4K5 levels in epididymal fat pads of mice were estimated by immunoblotting (panel i). Histone $\mathrm{H} 3$ was used as loading control The fold change of quantified band intensity is graphically represented in panel (ii) and (iii); ;n=4 in each group; one-way ANOVA with Dunnett's multiple comparision: ${ }^{*} P<0.05$, ${ }^{* *} P<0.01$, ${ }^{* * *} P<0.001$, ns: not significant. (f) The presence of LTK-14A in the physiological system upon administering the compound was verified by LC-ESI-MS analysis of the metabolite extracts of liver. 


\section{Figure 8}

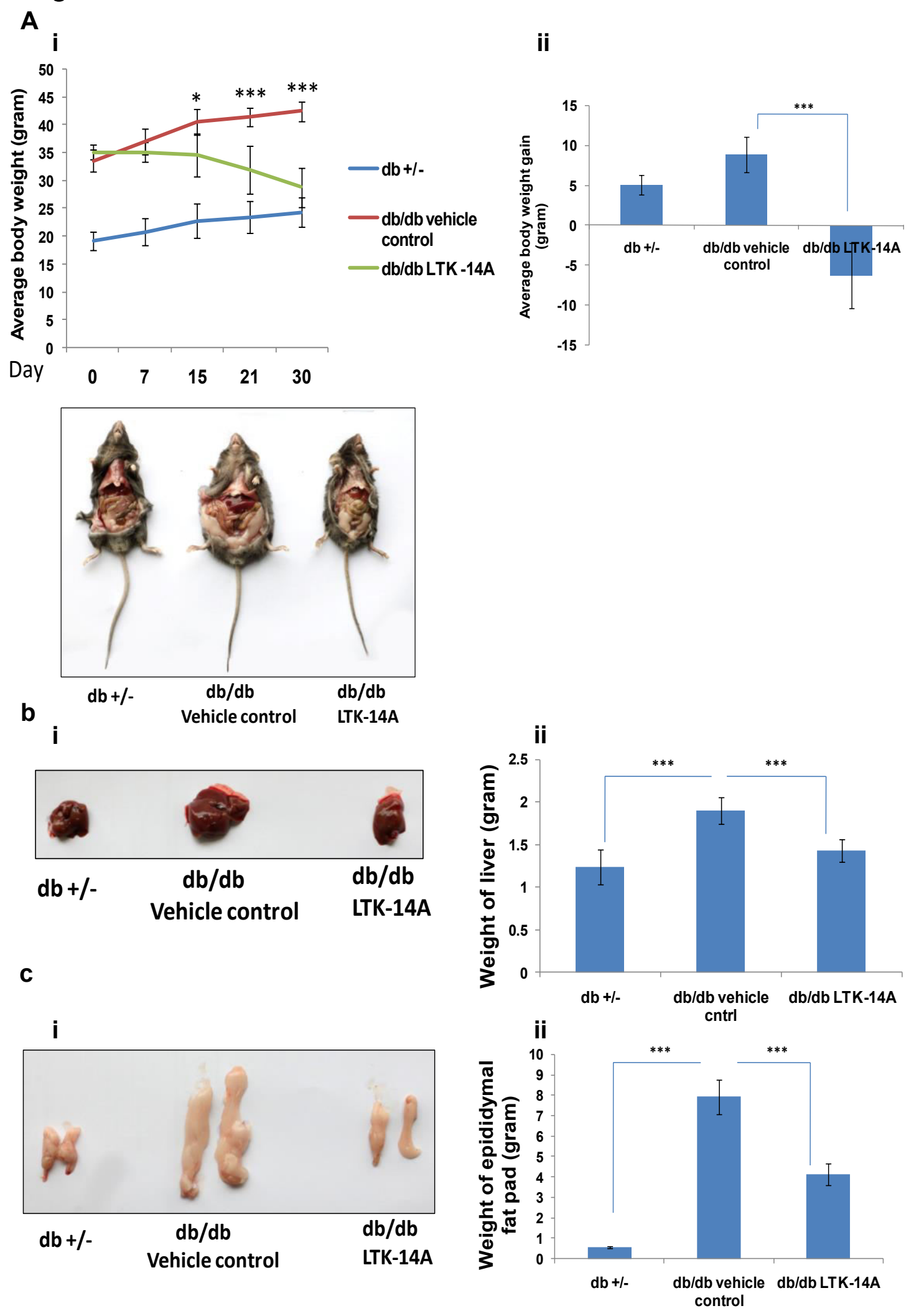




\section{Figure 8 (..continued)}
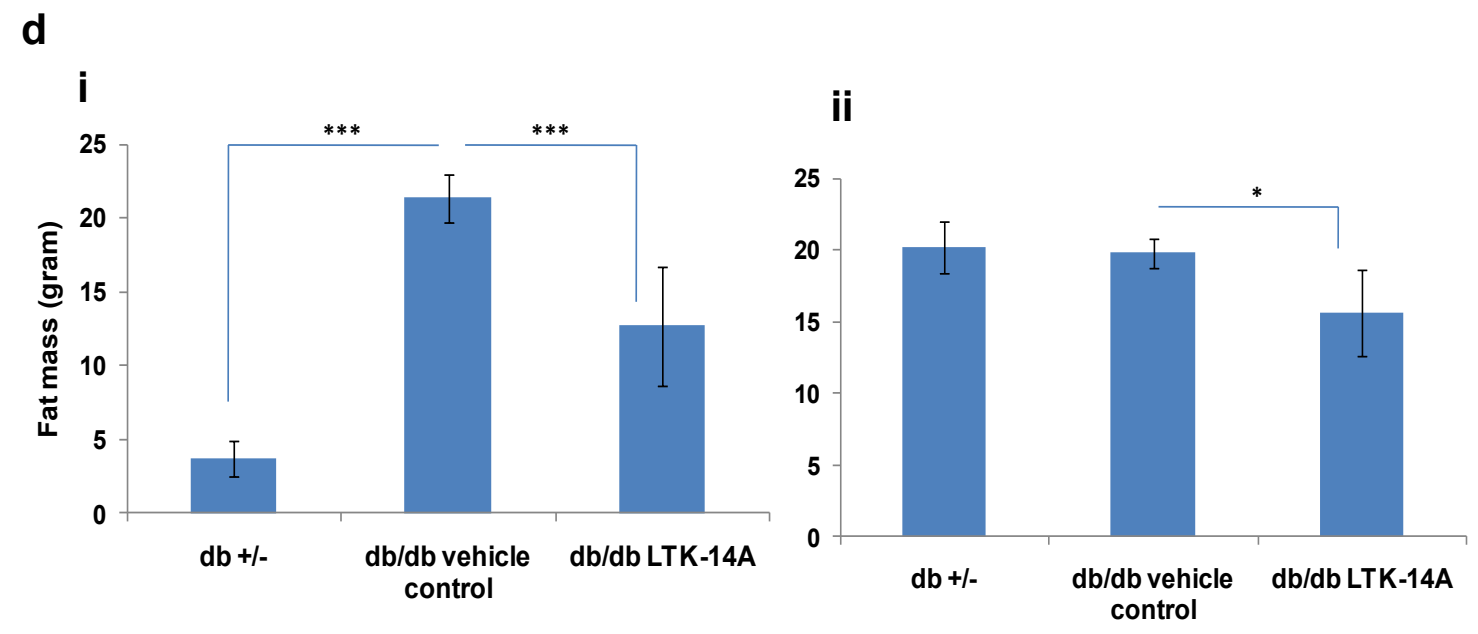

e. i

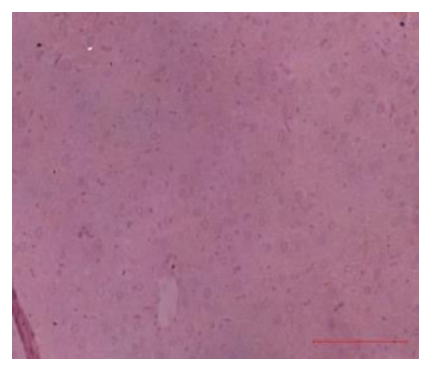

$\mathrm{db}+/-$

ii

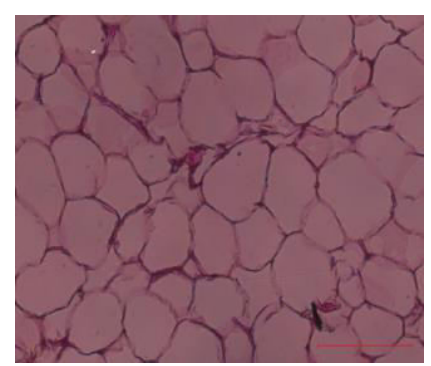

$d b+/-$

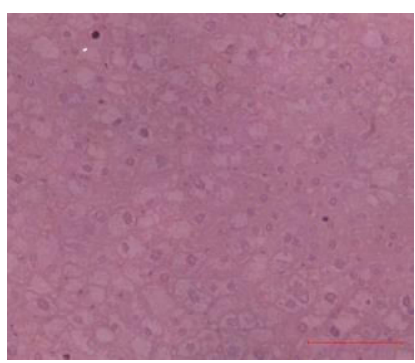

$\mathrm{db} / \mathrm{db}$ vehicle control

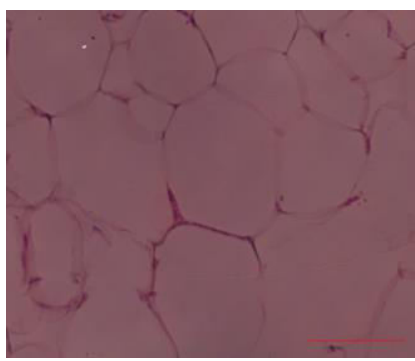

$\mathrm{db} / \mathrm{db}$ vehicle control

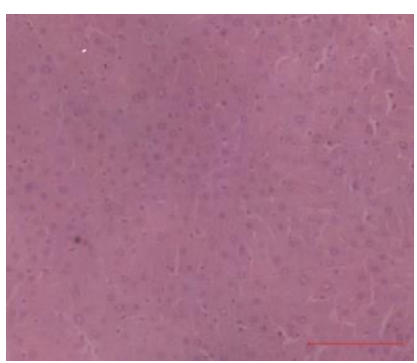

$\mathrm{db} / \mathrm{db}$ LTK-14A

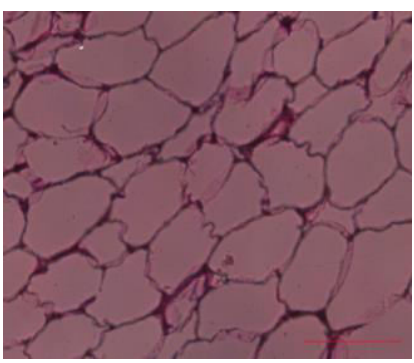

$\mathrm{db} / \mathrm{db}$ LTK-14A 
Figure 8 (..continued)

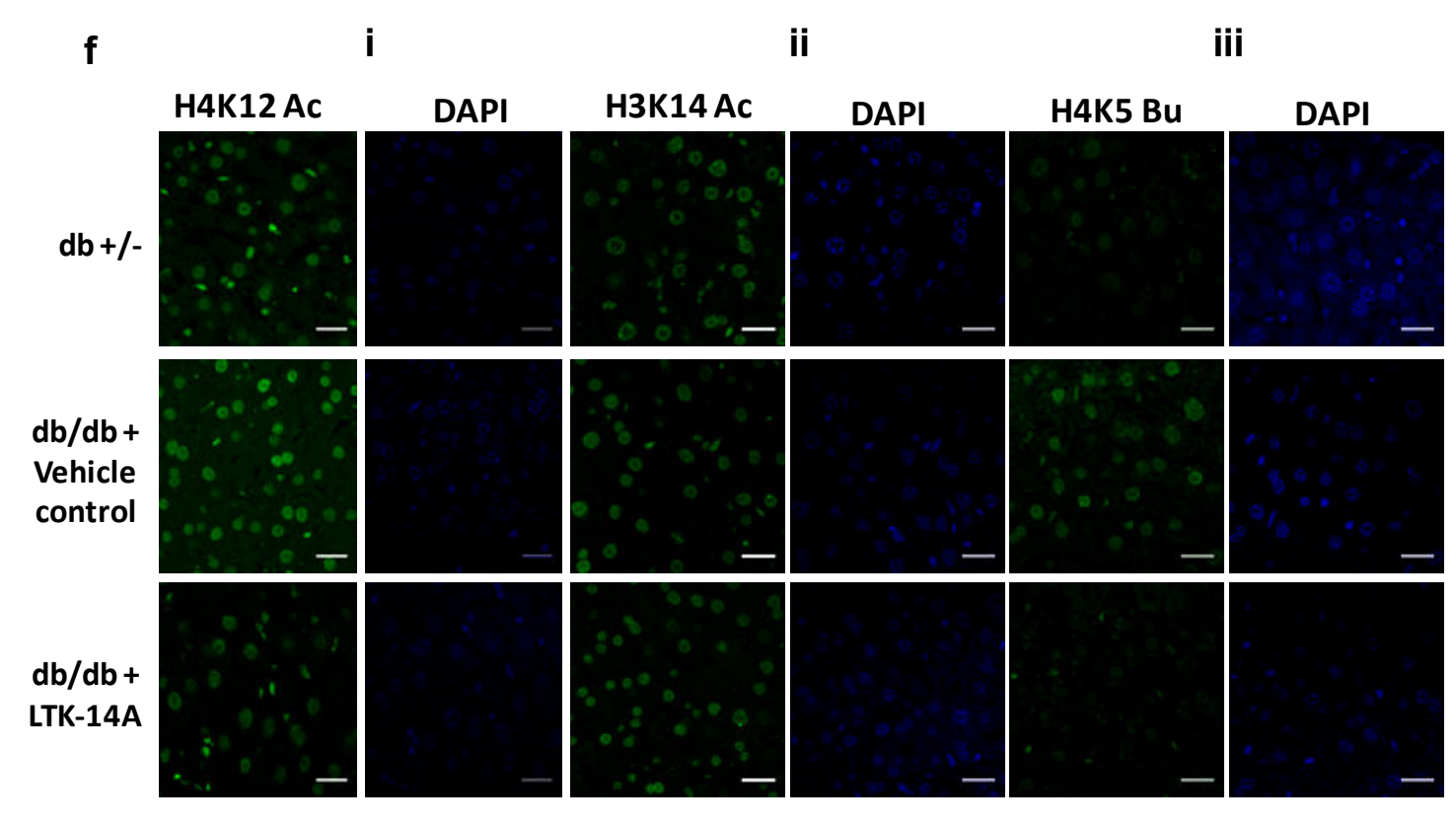

iv

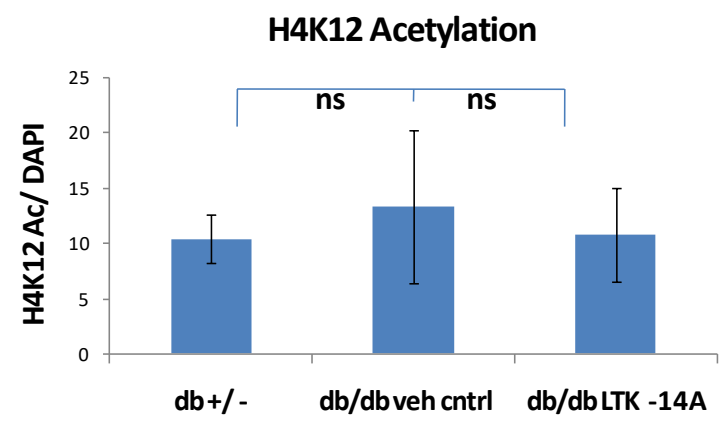

v

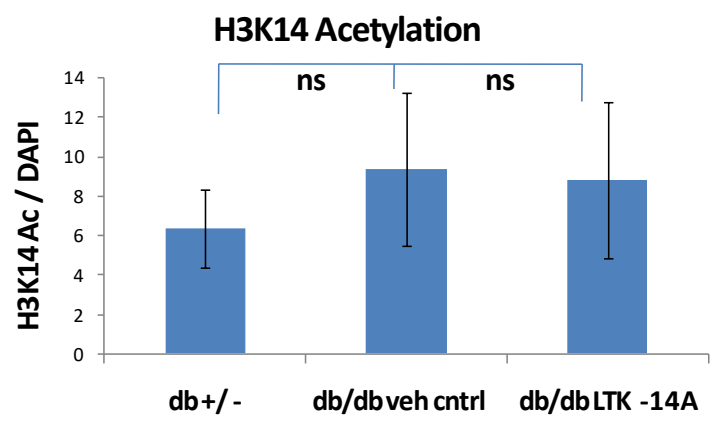

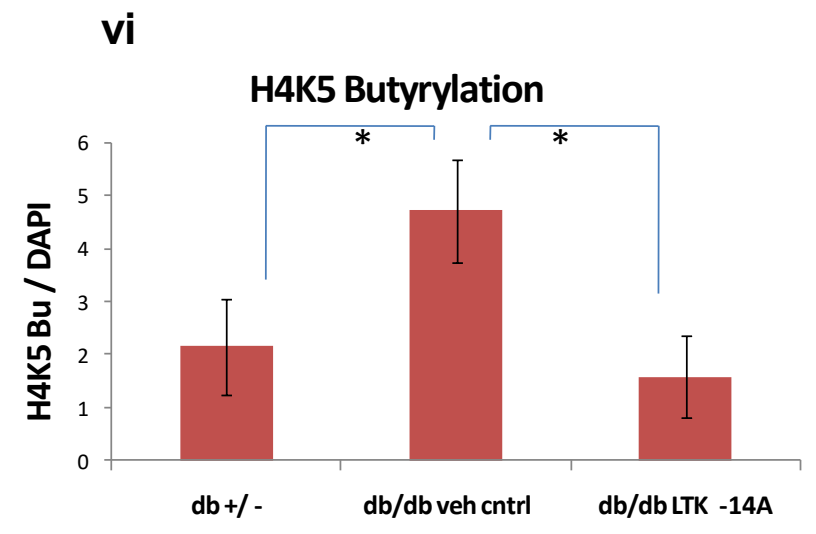




\section{Figure 8 (..continued)}

g
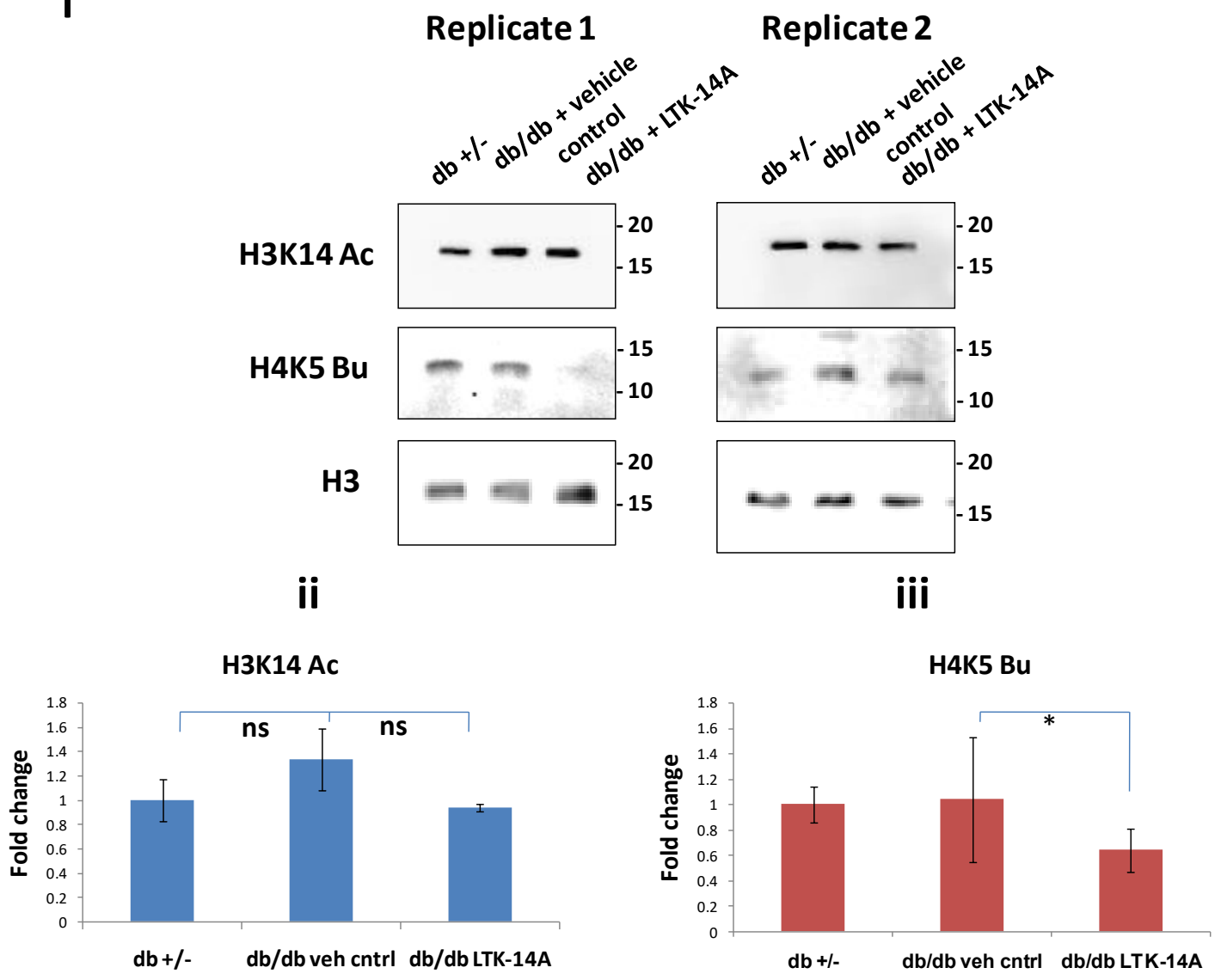
bioRxiv preprint doi: https://doi.org/10.1101/2021.08.01.454641; this version posted August 1, 2021. The copyright holder for this preprint (which was not certified by peer review) is the author/funder. All rights reserved. No reuse allowed without permission.

Fig. 8: Oral gavaging of the butyrylation inhibitor reduces weight of genetically obese $\mathrm{db} / \mathrm{db}$ mice: (a) Trend of weight gaining (panel i) and average body weight gained after 30 days (panel ii) of $\mathrm{db}+/-$ and $\mathrm{db} / \mathrm{db}$ mice orally administered with either LTK-14A or vehicle solution; $n=6$ to 7 in each group.

Representative images of dissected bodies showing fat accumulation (panel iii) of the mice from three groups. (b) Representative images of liver (panel i) from the mice of three groups and average weight of liver (panel ii) depicted graphically; $n=6$ to 7 in each group. (c) Representative images of epididymal fat pads (panel i) from the mice of three groups and average weight of fat pads (panel ii) depicted graphically; ; $n=6$ to 7 in each group (d) Total fat mass (panel i) and total lean mass (panel ii) of mice from three groups are depicted graphically; $n=6$ to 7 in each group. (e) Representative images of morphology of liver (panel i) and adipose tissue (panel ii) from the mice of the three groups, as studied by hematoxylin and eosin staining. (f) Immunofluorescence microscopy of liver sections stained with antibodies against histone H4K12 acetylation (panel i), histone H3K14 acetylation (panel ii) and histone H4K5 butyrylation (panel iii). The fluorescence intensity of the modified histone marks was normalised against DAPI staining of whole nuclei for quantitation which is graphically depicted in panel iv, $v$ and vi ;n=4 in each group; one-way ANOVA with Dunnett's multiple comparision: ${ }^{*} \mathrm{P}<0.05$, ${ }^{* *} \mathrm{P}<0.01$, ${ }^{* * *} \mathrm{P}<$ 0.001, ns: not significant. (g) Histone H3K14 acetylation and H4K5 levels in epididy mal fat pads of mice were estimated by immunoblotting (panel i). Histone $\mathrm{H} 3$ was used as loading control The fold change of quantified band intensity is graphically represented in panel (ii) and (iii); $n=4$ in each group; one-way ANOVA with Dunnett's multiple comparision: ${ }^{*} P<0.05$, ${ }^{* *} \mathrm{P}<0.01$, ${ }^{* * *} \mathrm{P}<0.001$, ns: not significant. 


\section{Graphical abstract}

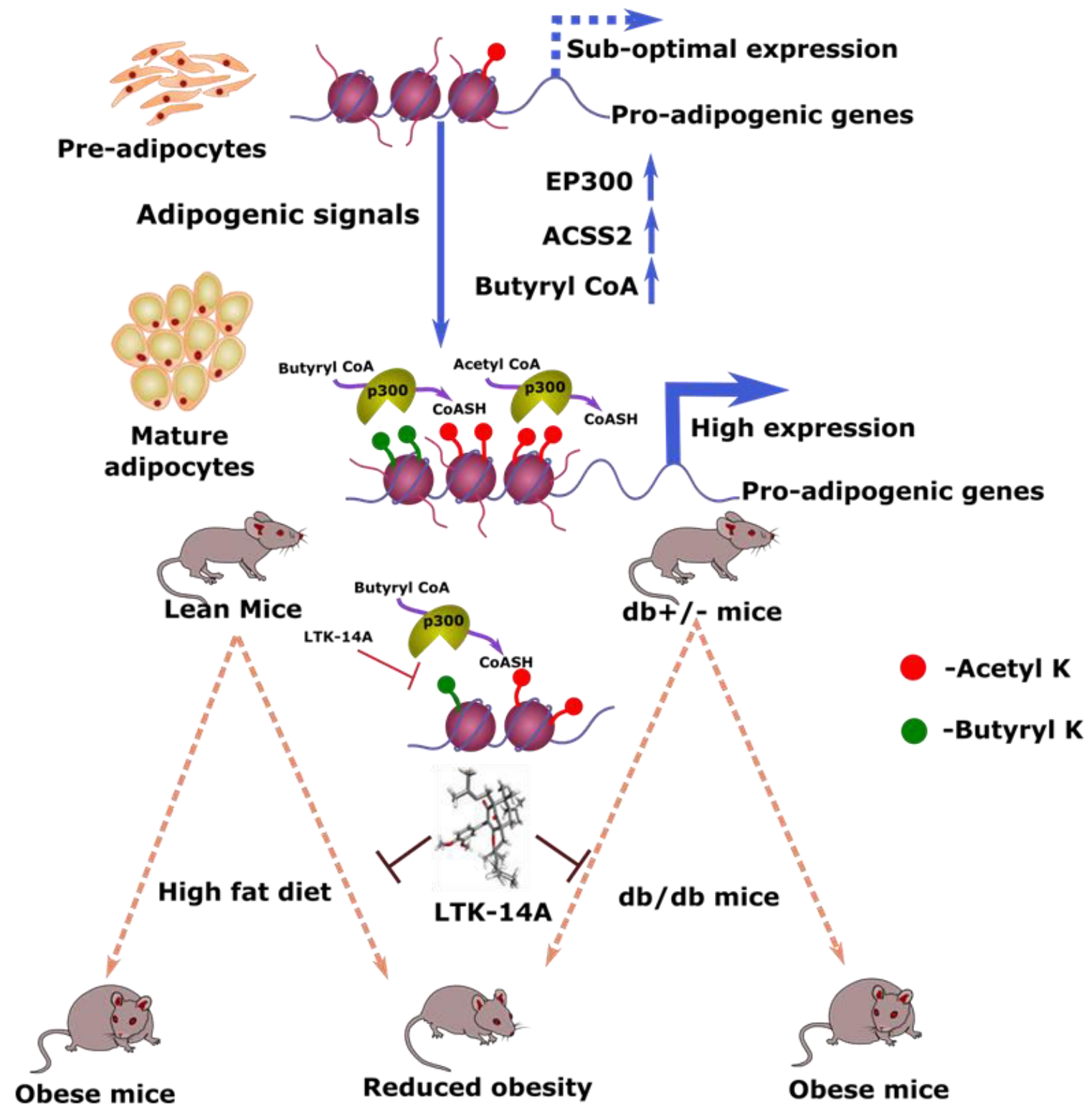




\section{Graphical abstract :}

\section{Proposed model for the role of $\mathrm{p} 300$-mediated histone butyrylation in adipogenesis:}

In pre-adipocytes, there exists a basal level of histone acetylation while butyrylation is present to a much lesser extent owing to low stoichiometric levels of butyryl CoA. Induction of adipogenesis causes a simultaneous upregulation of histone acetylation and butyrylation marks leading to increased rate of adipogenesis and concomitant transcriptional activation of pro-adipogeneic genes.

Onset of obesity in mice, either due to excess energy intake through high fat diet consumption or increased de novo synthesis of fatty acids due to leptin receptor gene mutation leading to hyperphagic behavior, is accompanied by adipocyte hyperplasia and hypertrophy. Both the organs of adipose tissue and liver found to have enhanced levels of H4K5 butyrylation during obesity. LTK-14A, a butyrylation specific inhibitor could efficiently prevent the processes of adipogenesis and adipocyte hypertrophy due to inhibition of H4K5 butyrylation in these organs. Thus the compound could attenuate weight gain by selective inhibition of butyrylation without affecting acetylation, thereby highlighting the importance of histone butyrylation in adipogenesis and obesity. 